Canad. J. Math. Vol. 71 (3), 2019 pp. 501-532

http://dx.doi.org/10.4153/CJM-2017-055-8

() Canadian Mathematical Society 2018

\title{
Isomorphic Structure of Cesàro and Tandori Spaces
}

\author{
Sergey V. Astashkin, Karol Lesnik, and Lech Maligranda
}

\begin{abstract}
We investigate the isomorphic structure of the Cesàro spaces and their duals, the Tandori spaces. The main result states that the Cesàro function space $\mathrm{Ces}_{\infty}$ and its sequence counterpart $\operatorname{ces}_{\infty}$ are isomorphic. This is rather surprising since $\mathrm{Ces}_{\infty}$ (like Talagrand's example) has no natural lattice predual. We prove that $\operatorname{ces}_{\infty}$ is not isomorphic to $\ell_{\infty}$ nor is $\operatorname{Ces}_{\infty}$ isomorphic to the Tandori space $\widetilde{L_{1}}$ with the norm $\|f\|_{\widetilde{L}_{1}}=\|\widetilde{f}\|_{L_{1}}$, where $\widetilde{f}(t):=\operatorname{ess~sup}_{s \geq t}|f(s)|$. Our investigation also involves an examination of the Schur and Dunford-Pettis properties of Cesàro and Tandori spaces. In particular, using results of Bourgain we show that a wide class of Cesàro-Marcinkiewicz and Cesàro-Lorentz spaces have the latter property.
\end{abstract}

\section{Introduction and Contents}

The classical Cesàro spaces most commonly appear as optimal domains of the Cesàro (Hardy) operator or some of its versions (see [DS07,NP10,LM15b]; see also [ORSP08, CR16, CR17], where the vector measures' point of view is presented). Moreover, they can coincide with the so-called down spaces, which were introduced and investigated by Sinnamon [KMS07, MS06, Si94, Si01, Si07], but have their roots in the papers of Halperin and Lorentz. In comparison to the function case, there is a much richer literature devoted to Cesàro sequence spaces and their duals (see the classical paper of Bennett [Be96] and also [CH01, CMP00, Ja74, KK12, MPS07]). Development of this topic related to the weighted case, including the so-called blocking technique, can be found in [GE98]. In this paper we investigate the isomorphic structure of abstract Cesàro spaces $C X$ and their duals, Tandori spaces $\widetilde{X}$, on three separable measure spaces $\mathbb{N},[0, \infty)$ and $[0,1]$. For a Banach ideal space $X$ of measurable functions on $I=[0, \infty)$ or $I=[0,1], C X$ is defined as the space of all measurable functions $f$ on $I$ such that $C|f| \in X$, equipped with the norm $\|f\|_{C X}:=\|C|f|\|_{X}$, where $C$ denotes the Cesàro operator, i.e., $(C f)(x):=\frac{1}{x} \int_{0}^{x} f(t) d t$ for $0<x \in I$. For a Banach ideal sequence space $X$, one uses the corresponding discrete Cesàro operator $\left(C_{d} x\right)_{n}:=\frac{1}{n} \sum_{k=1}^{n} x_{k}, n \in \mathbb{N}$ in the definition of the Cesàro space .

The study of abstract Cesàro function spaces, under this name, started in [LM15a], where a description of their Köthe duals by the so-called Tandori spaces can be found. It is worth noting here that the results obtained differ substantially in the

Received by the editors August 21, 2017; revised November 24, 2017.

Published electronically April 6, 2018.

Author S. V. A. was partially supported by the Ministry of Education and Science of the Russian Federation (project 1.470.2016/1.4) and author K. L. was partially supported by the grant 04/43/DSPB/0086 from the Polish Ministry of Science and Higher Education.

AMS subject classification: 46E30, 46B20, 46B42, 46B45.

Keywords: Cesàro and Tandori sequence spaces, Cesàro and Tandori function spaces, Cesàro operator, Banach ideal space, symmetric space, Schur property, Dunford-Pettis property, isomorphism. 
cases $I=[0, \infty)$ and $I=[0,1]$. Continuing the same direction of research, the authors examined interpolation properties of these spaces [LM16]. Investigation of the isomorphic structure of classical Cesàro function spaces $\operatorname{Ces}_{p}:=C L_{p}$ was initiated in [AM09] (see also [AM14]); however, studying classical Cesàro sequence spaces $\operatorname{ces}_{p}:=C \ell_{p}$ started much earlier (see [MPS07] and the references therein). Among other things, the existence of an isomorphism between the spaces $\operatorname{Ces}_{p}[0, \infty)$ and $\operatorname{Ces}_{p}[0,1]$ for all $1<p \leq \infty$ has been proved [AM09]. On the other hand, $\operatorname{Ces}_{p}(I)$ and ces $p$ for any $1<p<\infty$ are clearly not isomorphic because, in contrast to ces $p$, the space $\operatorname{Ces}_{p}(I)$ is not reflexive.

Therefore, the only remaining question (already formulated in [AM09, AM14]) was whether or not $\mathrm{Ces}_{\infty}$ is isomorphic to $\operatorname{ces}_{\infty}$. Theorem 5.1, one of the main results of the present paper, answers this problem in the affirmative. It is instructive to compare this result with the well-known Pełczyński theorem on the existence of an isomorphism between the spaces $L_{\infty}$ and $\ell_{\infty}$ [Pe58] and also with Leung's result that showed, using Pełczyński decomposition method, that the weak $L_{p}$-spaces: $L_{p, \infty}[0,1], L_{p, \infty}[0, \infty)$, and $\ell_{p, \infty}$ for every $1<p<\infty$ are all isomorphic [Le93].

On the other hand, we prove that $\operatorname{ces}_{\infty}$ and $\operatorname{Ces}_{\infty}(I)$ are not isomorphic to $\ell_{\infty}$ or to the Tandori space $\widetilde{L_{1}}(I)$ with the norm $\|f\|_{\widetilde{L}_{1}}=\|\widetilde{f}\|_{L_{1}}$, where

$$
\widetilde{f}(t):=\underset{s \in I, s \geq t}{\operatorname{ess} \sup }|f(s)| \text {. }
$$

Moreover, if $X$ is a reflexive symmetric space on $[0,1]$ and the Cesàro operator $C$ is bounded on $X$, then $C X$ is not isomorphic to any symmetric space on $[0,1]$. The main tool in proving these results comes from the fact that an arbitrary Cesàro space $C X$ (and, in particular, $\operatorname{ces}_{\infty}$ and $\operatorname{Ces}_{\infty}(I)$ ) contains a complemented copy of $L_{1}[0,1]$, provided the Cesàro operator $C$ is bounded in $X$ (see Proposition 2.2), but the other ones do not. We also make use of a characterization due to Hagler-Stegall [HS73] of dual Banach spaces containing complemented copies of $L_{1}[0,1]$; the Cembranos-Mendoza result [CM08], stated that the mixed-norm space $\ell_{\infty}\left(\ell_{1}\right)$ contains a complemented copy of $L_{1}[0,1]$, while the space $\ell_{1}\left(\ell_{\infty}\right)$ does not.

In this paper, along with the isomorphic structure of abstract Cesàro and Tandori spaces we also study their Schur and Dunford-Pettis properties (being isomorphic invariants). In particular, we are able to find a new rather natural Banach space nonisomorphic to $\ell_{1}$ with the Schur property, namely, the sequence Tandori space $\widetilde{\ell}_{1}$ with the norm $\left\|\left(a_{k}\right)\right\|_{\widetilde{\ell_{1}}}=\left\|\left(\widetilde{a_{k}}\right)\right\|_{\ell_{1}}$, where $\widetilde{a_{k}}:=\sup _{i \geq k}\left|a_{i}\right|$. Regarding the Dunford-Pettis property, we note that, generally, it is not easy to determine whether a given space has this property. We apply here a deep result of Bourgain [Bo81] that shows that every $\ell_{\infty}$-sum of $L_{1}$-spaces has the Dunford-Pettis property. Using this, Bourgain also deduced that the spaces of vector-valued functions $L_{1}(\mu, C(K))$ and $C\left(K, L_{1}(\mu)\right)$ and their duals, where $\mu$ and $K$ are a $\sigma$-finite measure and any compact Hausdorff set, respectively, have the Dunford-Pettis property. Using these facts and a suitable description of Cesàro and Tandori spaces obtained in this paper, we will prove that $\operatorname{Ces}_{\infty}(I), \widetilde{L_{1}}(I)$ and, under some conditions on dilation indices of a function $\varphi$, Cesàro-Marcinkiewicz spaces $C M_{\varphi}[0, \infty)$, their separable parts $C\left(M_{\varphi}^{0}\right)(I)$, CesàroLorentz spaces $C \Lambda_{\varphi}(I)$ as well as Tandori-Lorentz spaces $\widetilde{\Lambda_{\varphi}}(I)$ (in both cases $I=$ $[0, \infty)$ and $I=[0,1])$ all enjoy the Dunford-Pettis property. Recall that Kamińska and 
Mastyło [KM00] proved that $\ell_{1}, c_{0}$ and $\ell_{\infty}$ are the only symmetric sequence spaces with the Dunford-Pettis property and there exist exactly six non-isomorphic symmetric spaces on $[0, \infty)$ enjoying the latter property: $L_{1}, L_{\infty}, L_{1} \cap L_{\infty}, L_{1}+L_{\infty}$, and the closures of $L_{1} \cap L_{\infty}$ in both $L_{\infty}$ and $L_{1}+L_{\infty}$. The paper is organized as follows. Following this Introduction, Section 2 collects some necessary preliminaries, first, on Banach ideal and symmetric spaces and then, on Cesàro and Tandori spaces. Here, we recall also Theorem 2.1 related to the duality from [LM15a] and prove Proposition 2.2 on the existence of a complemented copy of $L_{1}[0,1]$ in an arbitrary Cesàro space $C X$ provided the Cesàro operator $C$ is bounded in $X$. These results are frequently used throughout the paper.

Section 3 contains results related to studying the Schur and Dunford-Pettis properties of Tandori and Cesàro sequence spaces. We prove that $\widetilde{\ell_{1}}$ has the Schur property (Theorem 3.1) and ces $s_{\infty}$ contains a complemented copy of $L_{1}[0,1]$ (Theorem 3.7). Moreover, we investigate the conditions under which both Cesàro-Marcinkiewicz and Cesàro-Lorentz sequence spaces as well as their duals have the Dunford-Pettis property (see Theorems 3.8 and 3.9). Finally, we show that the spaces $C X$ and $\widetilde{X}$ fail to have the Dunford-Pettis property whenever a symmetric sequence space $X$ is reflexive and the discrete Cesàro operator is bounded in $X$ or in $X^{\prime}$, respectively (Theorem 3.11).

Section 4 deals with the Dunford-Pettis property of Cesàro and Tandori function spaces. It is proved that, under the assumption $q_{\varphi}<1$, both Tandori-Lorentz space $\widetilde{\Lambda_{\varphi}}[0, \infty)$ and Cesàro-Marcinkiewicz space $C M_{\varphi}[0, \infty)$ have the DunfordPettis property (Theorem 4.1). In particular, two non-isomorphic spaces $\operatorname{Ces}_{\infty}(I)$ and $\widetilde{L_{1}}(I)$ have the latter property (see Theorem 4.3). A similar result also holds for the separable parts of the Cesàro-Marcinkiewicz spaces $C M_{\varphi}[0, \infty)$ and $C M_{\varphi}[0,1]$ provided $\lim _{t \rightarrow 0^{+}} \varphi(t)=0$ and $q_{\varphi}<1$ or $q_{\varphi}^{0}<1$, respectively (Theorems 4.6 and 4.8). Moreover, if $X$ is a reflexive symmetric function space satisfying some conditions, then $C X$ and $\widetilde{X}$ fail to have the Dunford-Pettis property (Theorem 4.9).

Section 5, contains one of the main results of the paper, showing that the spaces $\mathrm{Ces}_{\infty}$ and $\operatorname{ces}_{\infty}$ are isomorphic (Theorem 5.1). This gives a positive answer to the question posed in [AM09, Problem 1] and repeated in [AM14, Problem 4]. An interesting consequence of this result is the fact that the space $\mathrm{Ces}_{\infty}$ is isomorphic to a dual space, although $\left[\left(\operatorname{Ces}_{\infty}\right)^{\prime}\right]^{0}=\left(\widetilde{L_{1}}\right)^{0}=\{0\}$ (Corollary 5.2) and so there is no natural candidate for its predual. For ces $\infty$, however, the predual is $\widetilde{\ell_{1}}$, because $\left(\widetilde{\ell_{1}}\right)^{*}=\left(\widetilde{\ell_{1}}\right)^{\prime}=$ ces $_{\infty}$. Observing that this phenomenon has its counterpart in the general theory of Banach lattices, we discuss its relation to Lotz's result [Lo75] and to Talagrand's example of a separable Banach lattice being a dual space (and hence having the Radon-Nikodym property) such that for each $x^{*} \in E^{*}$, the interval $\left[0,\left|x^{*}\right|\right]$ is not weakly compact [Ta81] (see Proposition 5.4). Finally, we prove that $\operatorname{Ces}_{\infty}(I)$ is isomorphic to the space $\left(\oplus_{k=1}^{\infty} \mathcal{M}[0,1]\right)_{\ell_{\infty}}$, where $\mathcal{M}[0,1]$ is the space of regular Borel measures on $[0,1]$ of finite variation (Theorem 5.5). 


\section{Definitions and Basic Facts}

\subsection{Banach Ideal Spaces and Symmetric Spaces}

By $L^{0}=L^{0}(I)$ we denote the set of all equivalence classes of real-valued Lebesgue measurable functions defined on $I=[0,1]$ or $I=[0, \infty)$. A Banach ideal space $X=$ $(X,\|\cdot\|)$ on $I$ is understood as a Banach space contained in $L^{0}(I)$ that satisfies the so-called ideal property: if $f, g \in L^{0}(I),|f| \leq|g|$ almost everywhere with respect to the Lebesgue measure on $I$ and $g \in X$, then $f \in X$ and $\|f\| \leq\|g\|$. Sometimes we write $\|\cdot\|_{X}$ to be sure which norm in the space is considered. If not stated otherwise, we suppose that a Banach ideal space $X$ contains a function $f \in X$ with $f(x)>0$ almost everywhere on $I$ (such a function is called the weak unit in $X$ ), which implies that supp $X=I$. Similarly we define a Banach ideal sequence space, i.e., on $I=\mathbb{N}$ with the counting measure.

Since an inclusion of two Banach ideal spaces is continuous, we prefer to write in this case $X \hookrightarrow Y$ rather than $X \subset Y$. Moreover, the symbol $X \stackrel{A}{\rightarrow} Y$ indicates that $X \hookrightarrow Y$ with the norm of the inclusion operator not bigger than $A$, i.e., $\|f\|_{Y} \leq A\|f\|_{X}$ for all $f \in X$. Also, $X=Y$ (resp. $X \equiv Y$ ) means that the spaces $X$ and $Y$ have the same elements with equivalent (resp. equal) norms. By $X \simeq Y$ we denote the fact that the Banach spaces $X$ and $Y$ are isomorphic.

For a Banach ideal space $X=(X,\|\cdot\|)$ on $I$, the Köthe dual space (or associated space) $X^{\prime}$ is the space of all $f \in L^{0}(I)$ such that the associated norm

$$
\|f\|^{\prime}=\sup _{\substack{g \in X \\\|g\|_{X} \leq 1}} \int_{I}|f(x) g(x)| d x
$$

is finite. The Köthe dual $X^{\prime}=\left(X^{\prime},\|\cdot\|^{\prime}\right)$ is a Banach ideal space. Moreover, $X \stackrel{1}{\hookrightarrow} X^{\prime \prime}$ and we have equality $X=X^{\prime \prime}$ with $\|f\|=\|f\|^{\prime \prime}$ if and only if the norm in $X$ has the Fatou property, meaning that the conditions $0 \leq f_{n} \nearrow f$ almost everywhere on $I$ and $\sup _{n \in \mathbf{N}}\left\|f_{n}\right\|<\infty$ imply that $f \in X$ and $\left\|f_{n}\right\| \nearrow\|f\|$.

For a Banach ideal space $X=(X,\|\cdot\|)$ on $I$ with the Köthe dual $X^{\prime}$, the following generalized Hölder-Rogers inequality holds: if $f \in X$ and $g \in X^{\prime}$, then $f g$ is integrable and

$$
\int_{I}|f(x) g(x)| d x \leq\|f\|_{X}\|g\|_{X^{\prime}}
$$

A function $f$ in a Banach ideal space $X$ on $I$ is said to have an order continuous norm in $X$ if for any decreasing sequence of Lebesgue measurable sets $A_{n} \subset I$ with $m\left(\cap_{n=1}^{\infty} A_{n}\right)=0$, where $m$ is the Lebesgue measure, we have $\left\|f \chi_{A_{n}}\right\| \rightarrow 0$ as $n \rightarrow \infty$. The set of all functions in $X$ with order continuous norm is denoted by $X^{0}$. If $X^{0}=X$, then the space $X$ is said to be order continuous. For an order continuous Banach ideal space $X$, the Köthe dual $X^{\prime}$, and the dual space $X^{*}$ coincide. Moreover, a Banach ideal space $X$ with the Fatou property is reflexive if and only if both $X$ and its Köthe dual $X^{\prime}$ are order continuous.

For a weight $w(x)$, i.e., a measurable function on $I$ with $0<w(x)<\infty$ almost everywhere and for a Banach ideal space $X$ on $I$, the weighted Banach ideal space $X(w)$ 
is defined as the set $X(w)=\left\{f \in L^{0}: f w \in X\right\}$ with the norm $\|f\|_{X(w)}=\|f w\|_{X}$. Of course, $X(w)$ is also a Banach ideal space and $[X(w)]^{\prime} \equiv X^{\prime}(1 / w)$.

A Banach ideal space $X=\left(X,\|\cdot\|_{X}\right)$ is said to be a symmetric (or rearrangement invariant) space on $I$ if, from the conditions $f \in X, g \in L^{0}(I)$, and the equality $d_{f}(\lambda)=$ $d_{g}(\lambda)$ for all $\lambda>0$, where $d_{f}(\lambda):=m(\{x \in I:|f(x)|>\lambda\}), \lambda \geq 0$, it follows that $g \in X$ and $\|f\|_{X}=\|g\|_{X}$. In particular, $\|f\|_{X}=\left\|f^{*}\right\|_{X}$, where

$$
f^{*}(t)=\inf \left\{\lambda>0: d_{f}(\lambda)<t\right\}, t \geq 0 .
$$

For a symmetric function space $X$ on $I$, its fundamental function $\varphi_{X}$ is defined as

$$
\varphi_{X}(t)=\left\|\chi_{[0, t]}\right\|_{X}, \quad t>0,
$$

where $\chi_{E}$, throughout, will denote the characteristic function of a set $E$.

Let us recall some classical examples of symmetric spaces. Each increasing concave function $\varphi$ on $I, \varphi(0)=0$, generates the Lorentz space $\Lambda_{\varphi}$ (resp. Marcinkiewicz space $M_{\varphi}$ ) on $I$ endowed with the norm $\|f\|_{\Lambda_{\varphi}}=\int_{I} f^{*}(s) d \varphi(s)$, respectively,

$$
\|f\|_{M_{\varphi}}=\sup _{t \in I} \frac{\varphi(t)}{t} \int_{0}^{t} f^{*}(s) d s .
$$

In the case when $\varphi(t)=t^{1 / p}, 1<p<\infty$, the Marcinkiewicz space is also called the weak- $L_{p}$ space (briefly denoted by $L_{p, \infty}$ ) and the norm (2.2) is equivalent to the quasi-norm $\|f\|_{L_{p, \infty}}=\sup _{t \in I} t^{1 / p} f^{*}(t)$. In general, a space $M_{\varphi}$ is not separable (for example, when $\lim _{t \rightarrow 0^{+}} \frac{t}{\varphi(t)}=\lim _{t \rightarrow \infty} \frac{\varphi(t)}{t}=0$ ), but the spaces

$$
\left\{f \in M_{\varphi}: \lim _{t \rightarrow 0^{+}, \infty} \frac{\varphi(t)}{t} \int_{0}^{t} f^{*}(s) d s=0\right\} \text { in the case when } I=[0, \infty)
$$

and

$$
\left\{f \in M_{\varphi}: \lim _{t \rightarrow 0^{+}} \frac{\varphi(t)}{t} \int_{0}^{t} f^{*}(s) d s=0\right\} \text { in the case when } I=[0,1]
$$

with the norm (2.2) are the separable symmetric spaces which, in fact, coincide with the space $M_{\varphi}^{0}$ on $I=[0, \infty)$ or $I=[0,1]$, respectively, provided $\lim _{t \rightarrow 0^{+}} \varphi(t)=0$ [KPS82, pp. 115-116].

Let $\Phi$ be an increasing convex function on $[0, \infty)$ such that $\Phi(0)=0$. Denote by $L_{\Phi}$ the Orlicz space on $I$ [KR61, Ma89] endowed with the Luxemburg-Nakano norm $\|f\|_{L_{\Phi}}=\inf \left\{\lambda>0: \int_{I} \Phi(|f(x)| / \lambda) d x \leq 1\right\}$.

For a given symmetric space $X$ with the fundamental function $\varphi$ (recall that every such a function is equivalent to a concave function), we have $\Lambda_{\varphi} \stackrel{2}{\hookrightarrow} X \stackrel{1}{\rightarrow} M_{\varphi}$ and $\left(M_{\varphi}\right)^{\prime}=\Lambda_{\psi}$ with $\psi(t)=\frac{t}{\varphi(t)}, t>0$.

Similarly one can define Banach ideal and symmetric sequence spaces and all the above notions. In particular, the fundamental function of a symmetric sequence space $X$ is the function $\varphi_{X}(n)=\left\|\sum_{k=1}^{n} e_{k}\right\|_{X}, n \in \mathbb{N}$, where $\left\{e_{n}\right\}_{n=1}^{\infty}$ is the canonical basic sequence of $X$. Moreover, the Lorentz sequence space $\lambda_{\varphi}$ (resp. Marcinkiewicz sequence space $\left.m_{\varphi}\right)$ is defined as the space of all sequences $x=\left(x_{n}\right)_{n=1}^{\infty}$, for which the following norm is finite $\|x\|_{\lambda_{\varphi}}=\sum_{k=1}^{\infty} x_{k}^{*}(\varphi(k+1)-\varphi(k))$, respectively,

$$
\|x\|_{m_{\varphi}}=\sup _{n \in \mathbb{N}} \frac{\varphi(n)}{n} \sum_{k=1}^{n} x_{k}^{*}
$$


where $\varphi$ is an increasing concave function on $[0, \infty)$ and $\left(x_{k}^{*}\right)$ is the decreasing rearrangement of the sequence $\left(\left|x_{k}\right|\right)_{k=1}^{\infty}$. In the case when $\varphi(n)=n^{1 / p}$ for $1<p<\infty$, the Marcinkiewicz space $m_{\varphi}$ is also called the weak- $\ell_{p}$ space (denoted by $\ell_{p, \infty}$ for short) and the norm (2.3) is equivalent to the quasi-norm $\|x\|_{\ell_{p, \infty}}=\sup _{k \in \mathbb{N}} k^{1 / p} x_{k}^{*}$.

The dilation operators $\sigma_{s}(s>0)$ defined on $L^{0}(I)$ by $\sigma_{s} f(x)=f(x / s)$ if $I=[0, \infty)$ and $\sigma_{s} f(x)=f(x / s) \chi_{[0, \min (1, s)]}(x)$ if $I=[0,1]$ are bounded in any symmetric space $X$ on $I$ and $\left\|\sigma_{s}\right\|_{X \rightarrow X} \leq \max (1, s)$ [BS88, p. 148] and [KPS82, pp. 96-98]. These operators are also bounded in some Banach ideal spaces that are not symmetric. For example, if $X=L_{p}\left(x^{\alpha}\right)$, then $\left\|\sigma_{s}\right\|_{X \rightarrow X}=s^{1 / p+\alpha}$ (see [Ru80] for more examples). The Boyd indices of a symmetric space $X$ are defined by

$$
\alpha_{X}=\lim _{s \rightarrow 0^{+}} \frac{\ln \left\|\sigma_{s}\right\|_{X \rightarrow X}}{\ln s}, \quad \beta_{X}=\lim _{s \rightarrow \infty} \frac{\ln \left\|\sigma_{s}\right\|_{X \rightarrow X}}{\ln s},
$$

and we have $0 \leq \alpha_{X} \leq \beta_{X} \leq 1$ [KPS82, pp. 96-98], [LT79, p. 139].

For every $m \in \mathbb{N}$, let $\sigma_{m}$ and $\sigma_{1 / m}$ be the dilation operators defined in spaces of sequences $a=\left(a_{n}\right)$ by

$$
\begin{aligned}
\sigma_{m} a & =\left(\left(\sigma_{m} a\right)_{n}\right)_{n=1}^{\infty}=\left(a_{\left[\frac{m-1+n}{m}\right]}\right)_{n=1}^{\infty}=(\overbrace{a_{1}, a_{1}, \ldots, a_{1}}^{m}, \overbrace{a_{2}, a_{2}, \ldots, a_{2}}^{m}, \ldots), \\
\sigma_{1 / m} a & =\left(\left(\sigma_{1 / m} a\right)_{n}\right)_{n=1}^{\infty}=\left(\frac{1}{m} \sum_{k=(n-1) m+1}^{n m} a_{k}\right)_{n=1}^{\infty} \\
& =\left(\frac{1}{m} \sum_{k=1}^{m} a_{k}, \frac{1}{m} \sum_{k=m+1}^{2 m} a_{k}, \ldots, \frac{1}{m} \sum_{k=(n-1) m+1}^{n m} a_{k}, \ldots\right),
\end{aligned}
$$

[LT79, p. 131], [KPS82, p. 165]. They are bounded in any symmetric sequence space and also in some non-symmetric Banach ideal sequence spaces. For example,

$$
\left\|\sigma_{m}\right\|_{\ell_{p}\left(n^{\alpha}\right) \rightarrow \ell_{p}\left(n^{\alpha}\right)} \leq m^{1 / p} \max \left(1, m^{\alpha}\right), \quad\left\|\sigma_{1 / m}\right\|_{\ell_{p}\left(n^{\alpha}\right) \rightarrow \ell_{p}\left(n^{\alpha}\right)} \leq m^{-1 / p} \max \left(1, m^{-\alpha}\right) .
$$

The lower index $p_{\varphi}$ and upper index $q_{\varphi}$ of an arbitrary positive function $\varphi$ on $[0, \infty)$ are defined as

$$
p_{\varphi}=\lim _{s \rightarrow 0^{+}} \frac{\ln \bar{\varphi}(s)}{\ln s}, q_{\varphi}=\lim _{s \rightarrow \infty} \frac{\ln \bar{\varphi}(s)}{\ln s}, \text { where } \bar{\varphi}(s)=\sup _{t>0} \frac{\varphi(s t)}{\varphi(t)} .
$$

It is known [KPS82, Ma85, Ma89] that for a concave function $\varphi$ on $[0, \infty)$, we have $0 \leq p_{\varphi} \leq q_{\varphi} \leq 1$. Moreover, the estimate

$$
\int_{0}^{t} \frac{1}{\varphi(s)} d s \leq C \frac{t}{\varphi(t)} \quad \text { for all } t>0
$$

is equivalent to the condition $q_{\varphi}<1$ [KPS82, Lemma 1.4], [Ma85, Theorem 11.8], [Ma89, Theorem 6.4(a)]. If an increasing concave function $\varphi$ is defined on $[0,1]$ (resp. on $[1, \infty)$ ), then the corresponding indices $p_{\varphi}^{0}, q_{\varphi}^{0}\left(\right.$ resp. $\left.p_{\varphi}^{\infty}, q_{\varphi}^{\infty}\right)$ are the numbers defined as the limits in (2.4), where instead of $\bar{\varphi}$ we take the function $\bar{\varphi}^{0}(s)=$ $\sup _{0<t \leq \min (1,1 / s)} \varphi(s t) / \varphi(t)\left(\right.$ resp. $\left.\bar{\varphi}^{\infty}(s)=\sup _{t \geq \max (1,1 / s)} \varphi(s t) / \varphi(t)\right)$. Of course, $0 \leq p_{\varphi}^{0} \leq q_{\varphi}^{0} \leq 1$ (resp. $0 \leq p_{\varphi}^{\infty} \leq q_{\varphi}^{\infty} \leq 1$ ), and the estimate (2.5) for all $0<t \leq 1$ are equivalent to the condition $q_{\varphi}^{0}<1$. 
If $X_{n}, n=1,2, \ldots$, are Banach spaces and $1 \leq p \leq \infty$, then $\left(\oplus_{n=1}^{\infty} X_{n}\right) \ell_{p}$ is the Banach space of all sequences $\left\{x_{n}\right\}, x_{n} \in X_{n}, n=1,2, \ldots$, such that

$$
\left\|\left\{x_{n}\right\}\right\|:=\left(\sum_{n=1}^{\infty}\left\|x_{n}\right\|^{p}\right)^{1 / p}<\infty
$$

with natural modification in the case when $p$ is infinite.

For general properties of Banach ideal and symmetric spaces we refer to [BS88, KA77, KPS82, LT79, Ma89].

\subsection{Cesàro and Tandori Spaces}

The Cesàro and Copson operators $C$ and $C^{*}$ are defined, respectively, as

$$
\begin{aligned}
C f(x) & =\frac{1}{x} \int_{0}^{x} f(t) d t, \quad 0<x \in I, \\
C^{*} f(x) & =\int_{I \cap[x, \infty)} \frac{f(t)}{t} d t, \quad x \in I .
\end{aligned}
$$

By $\widetilde{f}$ we will understand the decreasing majorant of a given function $f$, i.e., $\widetilde{f}(x)=$ ess sup $_{t \in I, t \geq x}|f(t)|$. For a Banach ideal space $X$ on $I$ we define the abstract Cesàro space $C X=C X(I)$ as

$$
C X=\left\{f \in L^{0}(I): C|f| \in X\right\} \text { with the norm }\|f\|_{C X}=\|C|f|\|_{X},
$$

and the abstract Tandori space $\widetilde{X}=\widetilde{X}(I)$ as

$$
\widetilde{X}=\left\{f \in L^{0}(I): \widetilde{f} \in X\right\} \text { with the norm }\|f\|_{\widetilde{X}}=\|\widetilde{f}\|_{X} .
$$

In particular, if $X=L_{p}, 1<p \leq \infty$, we come to classical Cesàro spaces $\operatorname{Ces}_{p}=C L_{p}$, which were investigated in [AM08, AM09, AM13, AM14]. Note that the case when $p=1$ is not interesting here because it is easy to see that $\operatorname{Ces}_{1}[0, \infty)=\{0\}$ and $\operatorname{Ces}_{1}[0,1]=L_{1}\left(\ln \frac{1}{t}\right)$. The space $\operatorname{Ces}_{\infty}[0,1]$ appeared already in 1948 and is known as the Korenblyum-Krein-Levin space [KKL48, LZ66, Wn99].

It is clear that $\widetilde{X} \stackrel{1}{\rightarrow} X$ and $X \stackrel{A}{\rightarrow} C X$, provided $C$ is bounded on $X$ with $A=\|C\|_{X \rightarrow X}$. Moreover, if $X$ is a symmetric space on $I$, then for every $0<a<b, b \in I$ we have

$$
\left\|\chi_{[a, b]}\right\|_{\widetilde{X}}=\left\|\widetilde{\chi_{[a, b]}}\right\|_{X}=\left\|\chi_{[0, b]}\right\|_{X}=\varphi_{X}(b) .
$$

In the sequence case, the discrete Cesàro and Copson operators $C_{d}$ and $C_{d}^{*}$ are defined by $\left(C_{d} a\right)_{n}=\frac{1}{n} \sum_{k=1}^{n} a_{k}$ and $\left(C_{d}^{*} a\right)_{n}=\sum_{k=n}^{\infty} \frac{a_{k}}{k}, n \in \mathbb{N}$, and also the decreasing majorant $\widetilde{a}=\left(\widetilde{a_{n}}\right)$ of a given sequence $a=\left(a_{n}\right)$ is defined by $\widetilde{a_{n}}=\sup _{k \in \mathbb{N}, k \geq n}\left|a_{k}\right|$. Then the corresponding abstract Cesàro sequence space $C X$ and abstract Tandori sequence space $\widetilde{X}$ are defined as in (2.6) and (2.7). Again a lot of information is known about classical Cesàro sequence spaces $\operatorname{ces}_{p}:=C \ell_{p}, 1<p \leq \infty$ ([AM08, AM13, MPS07] and references given therein).

Abstract Cesàro and Copson spaces were investigated in [LM15a, LM15b], where the following results on their Köthe duality were proved [LM15a, Theorems 3, 5, 6]. 
Theorem 2.1 (i) If $X$ is a Banach ideal space on $I=[0, \infty)$ such that the Cesàro operator $C$ and the dilation operator $\sigma_{\tau}$ for some $\tau \in(0,1)$ are bounded on $X$, then $(C X)^{\prime}=\widetilde{X}^{\prime}$.

(ii) If $X$ is a symmetric space on $[0,1]$ with the Fatou property such that the operators $C, C^{*}: X \rightarrow X$ are bounded, then $(C X)^{\prime}=\widehat{X^{\prime}\left(w_{1}\right)}$, where $w_{1}(x)=\frac{1}{1-x}, x \in[0,1)$.

(iii) If $X$ is a Banach ideal sequence space such that the dilation operator $\sigma_{3}$ is bounded on $X^{\prime}$, then $(C X)^{\prime}=\widetilde{X}^{\prime}$. Moreover, in the extreme case when $X=L_{\infty}(I)$, the above duality results hold with the equality of norms.

The corresponding results on the Köthe duality of the classical spaces $\operatorname{Ces}_{p}(I)$ for $1<p<\infty$ were proved in [AM09] (see also [KK12]) showing a surprising difference among them in the cases $I=[0, \infty)$ and $I=[0,1]$. Much earlier, the identifications $\left(\operatorname{Ces}_{\infty}[0,1]\right)^{\prime} \equiv \widetilde{L_{1}}[0,1]$ and $\left(\operatorname{Ces}_{\infty}^{0}[0,1]\right)^{*} \equiv \tilde{L}_{1}[0,1]$ were obtained by Luxemburg and Zaanen [LZ66] and by Tandori [Ta55], respectively. A simple proof of the latter results both for $I=[0,1]$ and $I=[0, \infty)$ was given in [LM15a]. Moreover, according to Theorem 7 from [LM15a], if $w$ is a weight on $I$ such that $W(x)=\int_{0}^{x} w(t) d t<\infty$ for any $x \in I$, then setting $v(x)=x / W(x)$ we obtain

$$
\left(\operatorname{Ces}_{\infty}(v)\right)^{\prime}:=\left[C\left(L_{\infty}(v)\right)\right]^{\prime} \equiv \overline{L_{1}(w)} .
$$

A close identification for weighted Cesàro sequence spaces follows from a result by Alexiewicz [Al57], who showed that for a weight $w=\left(w_{n}\right)$ with $w_{n} \geq 0, w_{1}>0$, we have

$$
\left(\widetilde{l_{1}(w)}\right)^{\prime} \equiv \operatorname{ces}_{\infty}(v):=C\left(\ell_{\infty}(v)\right), \quad \text { where } v(n)=\frac{n}{\sum_{k=1}^{n} w_{k}}
$$

In particular, using the Fatou property of the space $\widetilde{\ell_{1}(w)}$, from (2.9) we infer

$$
\left(\operatorname{ces}_{\infty}(v)\right)^{\prime} \equiv\left(\widetilde{\ell_{1}(w)}\right)^{\prime \prime} \equiv \widetilde{\ell_{1}(w)} .
$$

In [LM15a, Theorem 1(d)], it was shown that if a Banach ideal space $X$ has the Fatou property, then the Cesàro and Tandori function spaces $C X$ and $\widetilde{X}$ also have it. Moreover, if a space $X$ is order continuous, then the Cesàro function space $C X$ is order continuous as well [LM16, Lemma 1]. However, the Tandori function space $\widetilde{X}$ is never order continuous [LM15a, Theorem 1(e)], which implies immediately that this space contains an isomorphic copy of $\ell_{\infty}$.

Next, we repeatedly make use of the following fact.

Proposition 2.2 If $X$ is a Banach ideal function space on $I=[0,1]$ or $I=[0, \infty)$ such that the operator $C$ is bounded on $X$, then $C X$ contains a complemented copy of $L_{1}[0,1]$. Moreover, if $\chi_{[0, a]} \in X$ for $0<a<1$, then $\widetilde{X} \neq\{0\}$ and $\widetilde{X}$ contains a complemented copy of $L_{\infty}[0,1]$.

Proof Suppose that $I=[0,1]$. Since $\operatorname{supp} X=I$ and the operator $C$ is bounded on $X$, then $\chi_{[a, 1]} \in X$ for any $0<a<1$. In fact, let $f_{0} \in X$ with $f_{0}(x)>0$ almost everywhere 
on $I$. Then $f_{0} \chi_{[0, a]} \in X$ and $\int_{0}^{a} f_{0}(t) d t=c>0$. Therefore, from the estimate

$$
\begin{aligned}
C\left(f_{0} \chi_{[0, a]}\right)(x) & \geq \frac{1}{x} \int_{0}^{x} f_{0}(t) \chi_{[0, a]}(t) d t \geq \frac{1}{x} \int_{0}^{x} f_{0}(t) \chi_{[0, a]}(t) d t \cdot \chi_{[a, 1]}(x), \\
& =\frac{1}{x} \int_{0}^{a} f_{0}(t) d t \cdot \chi_{[a, 1]}(x) \geq c \chi_{[a, 1]}(x), \quad 0<x \leq 1,
\end{aligned}
$$

and from the boundedness of $C$ on $X$ it follows that $\chi_{[a, 1]} \in X$. Now for $0<a<b<1$, one has

$$
\begin{aligned}
C\left(f \chi_{[a, b]}\right)(x) & =\frac{1}{x} \int_{a}^{x}|f(t)| d t \chi_{[a, b]}(x)+\frac{1}{x} \int_{a}^{b}|f(t)| d t \chi_{[b, 1]}(x) \\
& \leq \frac{1}{a} \int_{a}^{b}|f(t)| d t\left[\chi_{[a, b]}(x)+\chi_{[b, 1]}(x)\right]=\frac{1}{a}\|f\|_{L_{1}[a, b]} \cdot \chi_{[a, 1]}(x), \\
C\left(f \chi_{[a, b]}\right)(x) & =\frac{1}{x} \int_{a}^{x}|f(t)| \chi_{[a, b]}(t) d t \geq \frac{1}{x} \int_{a}^{x}|f(t)| \chi_{[a, b]}(t) d t \cdot \chi_{[b, 1]}(x) \\
& \geq \frac{1}{x} \int_{a}^{b}|f(t)| d t \cdot \chi_{[b, 1]}(x)=\|f\|_{L_{1}[a, b]} \cdot \chi_{[b, 1]}(x) .
\end{aligned}
$$

Thus,

$$
d\|f\|_{L_{1}[a, b]} \leq\left\|f \chi_{[a, b]}\right\|_{C X} \leq \frac{D}{a}\|f\|_{L_{1}[a, b]},
$$

where $d=\left\|\chi_{[b, 1]}\right\|_{X}$ and $D=\left\|\chi_{[a, 1]}\right\|_{X}$ are finite. Therefore, $\left.C X\right|_{[a, b]}=L_{1}[a, b]$ with equivalence of norms and, since $L_{1}[a, b] \simeq L_{1}[0,1]$ and the projection $P: f \mapsto f \chi_{[a, b]}$ is bounded in $C X$, the first claim of the proposition is proved if $I=[0,1]$. The case when $I=[0, \infty)$ can be treated in the same way, only the norm $\left\|\chi_{[b, 1]}\right\|_{X}$ should be replaced with $\left\|\frac{1}{x} \chi_{[b, \infty)}(x)\right\|_{X}$.

Regarding the space $\widetilde{X}$, we note that under the conditions imposed on $X$ by (2.8), we have $\overline{f \chi_{[a, b]}} \leq\|f\|_{L_{\infty}[a, b]} \cdot \overline{\chi_{[a, b]}}=\|f\|_{L_{\infty}[a, b]} \cdot \chi_{[0, b]}$ and conversely

$$
\overline{f \chi_{[a, b]}} \geq \overline{f \chi_{[a, b]}} \cdot \chi_{[0, a]}=\|f\|_{L_{\infty}[a, b]} \cdot \chi_{[0, a]},
$$

whence $\left\|\chi_{[0, a]}\right\|_{X}\|f\|_{L_{\infty}[a, b]} \leq\left\|f \chi_{[a, b]}\right\|_{\widetilde{X}} \leq\left\|\chi_{[0, b]}\right\|_{X}\|f\|_{L_{\infty}[a, b]}$. Thus, the image of the same projection $P f=f \chi_{[a, b]}$ is isomorphic to $L_{\infty}[0,1]$. Since $P$ is bounded, the proof is complete.

\section{On the Schur and Dunford-Pettis Properties of Cesàro and Tandori Sequence Spaces}

A Banach space $X$ is said to have the Dunford-Pettis property if, for all sequences $x_{n} \stackrel{w}{\rightarrow} 0$ in $X$ and $x_{n}^{*} \stackrel{w}{\rightarrow} 0$ in $X^{*}$, we have $\left\langle x_{n}^{*}, x_{n}\right\rangle \rightarrow 0$ as $n \rightarrow \infty$ or, equivalently, if any weakly compact operator $T: X \rightarrow Y$, where $Y$ is an arbitrary Banach space, is completely continuous, i.e., from $x_{n} \stackrel{w}{\rightarrow} 0$, it follows that $T\left(x_{n}\right)$ converges to 0 in the norm of $Y$. Examples of spaces satisfying the Dunford-Pettis property are $\ell_{1}, c_{0}, \ell_{\infty}, L_{1}(\mu), L_{\infty}(\mu)$ for every $\sigma$-finite measure $\mu$ and $C(K), C(K)^{*}=\mathcal{M}(K)$ for an arbitrary compact Hausdorff space $K$ [AK06, pp. 116-117], [Li04, pp. 57-67]). It is well known that infinite-dimensional reflexive spaces fail to have the DunfordPettis property. Moreover, if a dual space $X^{*}$ has the Dunford-Pettis property, then 
so does $X$ (the reverse implication is not true) and complemented subspaces of spaces with the Dunford-Pettis property also have it.

Recall that a Banach space $X$ has the Schur property if for any sequence $x_{n} \stackrel{w}{\rightarrow} 0$ in $X$ we have $\left\|x_{n}\right\| \rightarrow 0$ as $n \rightarrow \infty$ or, equivalently, if every weakly compact operator from $X$ to arbitrary Banach space $Y$ is compact. Of course, spaces with the Schur property have also the Dunford-Pettis property. Even though it has been known [Ba32, pp. 137139 ] that the space $\ell_{1}$ has the Schur property, only a few natural infinite-dimensional spaces enjoying it were found. See [Di80] and [Wn93] for a survey of results related to the Dunford-Pettis property and the Schur property, respectively, (see also [CG94]).

We start with proving the Schur property of the space $\widetilde{\ell_{1}}$. Note that $\widetilde{\ell_{1}}$ is not isomorphic to $\ell_{1}$. In fact, $\left\{\frac{1}{n} e_{n}\right\}$ is a normalized, unconditional basis in $\widetilde{\ell_{1}}$. On the other hand, $\ell_{1}$ has a unique unconditional structure, i.e., each normalized unconditional basis in $\ell_{1}$ is equivalent to $\left\{e_{n}\right\}$ [LT77, Theorem 2.b.9]. Therefore, if we assume that $\widetilde{\ell}_{1}$ is isomorphic to $\ell_{1}$, then $\left\{\frac{1}{n} e_{n}\right\}$ would be equivalent to $\left\{e_{n}\right\}$. But it is not the case, since we have both $\left\|\sum_{n=1}^{k} \frac{1}{n} e_{n}\right\|_{\widetilde{\ell}_{1}} \approx \ln k$ and $\left\|\sum_{n=1}^{k} e_{n}\right\|_{\ell_{1}}=k, k \in \mathbb{N}$.

\section{Theorem 3.1 The space $\widetilde{\ell_{1}}$ has the Schur property.}

Proof First, using (2.9) and the fact that $\widetilde{\ell}_{1}$ has an order continuous norm [LM16], we obtain $\left(\widetilde{\ell_{1}}\right)^{*}=\left(\widetilde{\ell_{1}}\right)^{\prime}=\operatorname{ces}_{\infty}$. Now let $\left\|x^{(n)}\right\|_{\widetilde{\ell}_{1}} \leq 1$ with $x^{(n)} \rightarrow 0$ weakly in $\widetilde{\ell_{1}}$ as $n \rightarrow \infty$. By the Banach-Alaoglu theorem the closed unit ball $B$ in ces $\infty$ is $w^{*}$-compact and metrizable, so, in particular, it is a $w^{*}$-complete metric space. For any $\varepsilon>0$ we put $B_{m}=\bigcap_{n \geq m}\left\{f \in B:\left|\left\langle f, x^{(n)}\right\rangle\right| \leq \varepsilon\right\}$. Then the sets $B_{m}$ are $w^{*}$-closed, $B_{1} \subset B_{2} \subset \cdots$, and $B=\bigcup_{m=1}^{\infty} B_{m}$. Thus, by the Baire theorem, there are $N, m_{1} \in \mathbb{N}, g=\left(g_{k}\right) \in B_{m_{1}}$, and $\delta>0$ such that $U:=\left\{f=\left(f_{k}\right) \in B:\left|f_{k}-g_{k}\right|<\delta, 1 \leq k \leq N\right\} \subset B_{m_{1}}$. Consequently, $U \subset B_{m}$ for each $m \geq m_{1}$. Fix $N_{1}>N$ such that

$$
\frac{\sum_{k=1}^{N}\left|g_{k}\right|}{N_{1}}<\varepsilon
$$

Clearly, the weak convergence of $\left\{x^{(n)}\right\}$ implies the coordinate convergence, so that there is $m_{2} \in \mathbb{N}$ such that for $n \geq m_{2}$

$$
\left\|x^{(n)} \chi_{\left[1, N_{1}\right)}\right\|_{\widetilde{\ell}_{1}} \leq \mathcal{\varepsilon} .
$$

For every $n \in \mathbb{N}$, there is $f^{(n)} \in B$ such that $\left\|x^{(n)} \chi_{\left[N_{1}, \infty\right)}\right\|_{\widetilde{\ell}_{1}}=\left\langle f^{(n)}, x^{(n)} \chi_{\left[N_{1}, \infty\right)}\right\rangle$. Without loss of generality, we may assume that $\operatorname{supp} f^{(n)} \subset\left[N_{1}, \infty\right)$. Setting

$$
g^{(n)}=g \chi_{[1, N)}+(1-\varepsilon) f^{(n)},
$$

we will show that $g^{(n)} \in U$ for all $n \in \mathbb{N}$. Since $g_{k}^{(n)}=g_{k}$ for each $1 \leq k \leq N$, it is enough to check only that $\left\|g^{(n)}\right\|_{\operatorname{ces}_{\infty}} \leq 1$. We have

$$
C\left|g^{(n)}\right|(j)= \begin{cases}C|g|(j) & \text { if } j<N, \\ \frac{1}{j} \sum_{k=1}^{N}\left|g_{k}\right| & \text { if } N \leq j<N_{1}, \\ \frac{1}{j}\left(\sum_{k=1}^{N}\left|g_{k}\right|+(1-\varepsilon) \sum_{k=N_{1}}^{j}\left|f_{k}^{(n)}\right|\right) & \text { if } j \geq N_{1} .\end{cases}
$$


Consequently, from (3.1) it follows that $C\left|g^{(n)}\right|(j) \leq 1$ for each $j \in \mathbb{N}$, i.e., $g^{(n)} \in U$ and therefore $g^{(n)} \in B_{m}$ if $m>m_{1}$. Finally, applying (3.2), for $n \geq m_{0}:=\max \left\{m_{1}, m_{2}\right\}$, we get

$$
\begin{aligned}
\left\|x^{(n)}\right\|_{\widetilde{\ell}_{1}} & \leq\left\|x^{(n)} \chi_{\left[1, N_{1}\right)}\right\|_{\widetilde{\ell}_{1}}+\left\|x^{(n)} \chi_{\left[N_{1}, \infty\right)}\right\|_{\widetilde{\ell}_{1}} \leq \varepsilon+\left|\sum_{k=N_{1}}^{\infty} x_{k}^{(n)} f_{k}^{(n)}\right| \\
& =\varepsilon+\left|\frac{1}{1-\varepsilon}\left((1-\varepsilon) \sum_{k=N_{1}}^{\infty} x_{k}^{(n)} f_{k}^{(n)}+\sum_{k=1}^{N} x_{k}^{(n)} g_{k}\right)-\frac{1}{1-\varepsilon} \sum_{k=1}^{N} x_{k}^{(n)} g_{k}\right| \\
& \leq \varepsilon+\frac{1}{1-\varepsilon}\left|\sum_{k=1}^{\infty} x_{k}^{(n)} g_{k}^{(n)}\right|+\frac{1}{1-\varepsilon}\left\|x^{(n)} \chi_{[1, N)}\right\|_{\widetilde{\ell}^{1}}\|g\|_{\operatorname{ces}_{\infty}} \\
& =\varepsilon+\frac{1}{1-\varepsilon}\left|\left\langle g^{n}, x^{n}\right\rangle\right|+\frac{1}{1-\varepsilon}\left\|x^{(n)} \chi_{[1, N)}\right\|_{\widetilde{\ell}^{1}}\|g\|_{\operatorname{ces}_{\infty} \leq \varepsilon+\frac{2 \varepsilon}{1-\varepsilon}}
\end{aligned}
$$

which shows that $\lim _{n \rightarrow \infty}\left\|x^{(n)}\right\|_{\widetilde{\ell}_{1}}=0$, as desired.

Corollary 3.2 The space ces ${ }_{\infty}^{0}$ has the Dunford-Pettis property.

Proof From (2.10) we have $\left(\operatorname{ces}_{\infty}^{0}\right)^{*}=\left(\operatorname{ces}_{\infty}^{0}\right)^{\prime}=\widetilde{\ell}_{1}$ and by the fact that a Banach space has the Dunford-Pettis property whenever its dual space has it, the result follows from Theorem 3.1.

Although the spaces $\widetilde{\ell}_{1}$ and $\ell_{1}$ are not isomorphic, $\ell_{1}$ is isomorphic to a subspace of $\widetilde{\ell_{1}}$ and so $\widetilde{\ell_{1}}$ can be treated as an extension of $\ell_{1}$ preserving the Schur property.

Theorem 3.3 The basic sequence $\left\{2^{-i} e_{2^{i}}\right\}_{i=0}^{\infty}$ is equivalent in the space $\widetilde{\ell_{1}}$ to the canonical $\ell_{1}$-basis.

Proof We prove that for all $n \in \mathbb{N}$ and $c_{i} \geq 0, i=0,1, \ldots, n$,

$$
\frac{1}{2} \sum_{i=0}^{n} c_{i} \leq\left\|\sum_{i=0}^{n} c_{i} 2^{-i} e_{2^{i}}\right\|_{\widetilde{\ell}_{1}} \leq \sum_{i=0}^{n} c_{i}
$$

Since $\left\|e_{m}\right\|_{\widetilde{\ell}_{1}}=m$, for every $m \in \mathbb{N}$, the right-hand side inequality is obvious. Thus, it is enough to check only the opposite inequality. If $c_{i} \geq 0$, then

$$
\begin{aligned}
\left(\sum_{i=0}^{n} c_{i} 2^{-i} e_{2^{i}}\right)^{\sim} & =\left(c_{0}, \frac{c_{1}}{2}, 0, \frac{c_{2}}{4}, 0,0,0, \frac{c_{3}}{8}, \ldots, \frac{c_{n-1}}{2^{n-1}}, 0, \ldots, \frac{c_{n}}{2^{n}}, 0 \ldots\right)^{\sim} \\
& =\left(c_{0}, \frac{c_{1}}{2}, \frac{c_{2}}{4}, \frac{c_{2}}{4}, \frac{c_{3}}{8}, \frac{c_{3}}{8}, \frac{c_{3}}{8}, \frac{c_{3}}{8}, \ldots, \frac{c_{n-1}}{2^{n-1}}, \frac{c_{n}}{2^{n}}, \ldots, \frac{c_{n}}{2^{n}}, \frac{c_{n}}{2^{n}}, 0 \ldots\right)^{\sim} \\
& \geq\left(c_{0}, \frac{c_{1}}{2}, \frac{c_{2}}{4}, \frac{c_{2}}{4}, \frac{c_{3}}{8}, \frac{c_{3}}{8}, \frac{c_{3}}{8}, \frac{c_{3}}{8}, \ldots, \frac{c_{n-1}}{2^{n-1}}, \frac{c_{n}}{2^{n}}, \ldots, \frac{c_{n}}{2^{n}}, \frac{c_{n}}{2^{n}}, 0 \ldots\right)
\end{aligned}
$$

so

$$
\left\|\sum_{i=0}^{n} c_{i} 2^{-i} e_{2^{i}}\right\|_{\widetilde{\ell}_{1}}=\left\|\left(\sum_{i=0}^{n} c_{i} 2^{-i} e_{2^{i}}\right)^{\sim}\right\|_{\ell_{1}} \geq c_{0}+\frac{1}{2} \sum_{i=1}^{n} c_{i} \geq \frac{1}{2} \sum_{i=0}^{n} c_{i} .
$$

Since the space $\widetilde{\ell_{1}}$ is order continuous, then from (2.9) it follows that $\left(\widetilde{\ell_{1}}\right)^{*}=$ $\left(\widetilde{\ell_{1}}\right)^{\prime}=\operatorname{ces}_{\infty}$. Therefore, taking into account that the space $\operatorname{ces}_{\infty}$ has the Fatou property, we obtain the following. 
Corollary 3.4 The basic sequence $\left\{2^{i} e_{2^{i}}\right\}_{i=0}^{\infty}$ is equivalent in the space $\operatorname{ces}_{\infty}$ to the canonical $c_{0}$-basis and $\ell_{\infty}$ is embedded into $\operatorname{ces}_{\infty}$.

Corollary 3.5 The space $\widetilde{\ell}_{1}$ is isomorphic to the space $\left(\oplus_{n=0}^{\infty} \ell_{\infty}^{2^{n}}\right) \ell_{1}$. Therefore, the space ces $c_{\infty}$ is isomorphic to the space $\left(\oplus_{n=0}^{\infty} \ell_{1}^{2^{n}}\right)_{\ell_{\infty}}$ and contains a complemented subspace isomorphic to $L_{1}[0,1]$.

Proof Let us define the linear operator $T$ from $\widetilde{\ell}_{1}$ to $\left(\oplus_{n=0}^{\infty} \ell_{\infty}^{2^{n}}\right) \ell_{1}$ as follows: if $c=$ $\left(c_{k}\right)_{k=1}^{\infty} \in \widetilde{\ell_{1}}$, then $T c=\left(d^{(n)}\right)_{n=0}^{\infty}$, where

$$
d^{(n)}=\left(d_{j}^{(n)}\right)_{j=1}^{2^{n}}, d_{j}^{(n)}:=\left(j-1+2^{n}\right) \cdot c_{j-1+2^{n}}, j=1,2, \ldots, 2^{n} .
$$

Assuming that $c_{k} \geq 0, k=1,2, \ldots$, by Theorem 3.3, we obtain

$$
\begin{aligned}
\|c\|_{\widetilde{\ell_{1}}} & =\left\|\sum_{k=0}^{\infty} c_{k} e_{k}\right\|_{\widetilde{\ell}_{1}}=\left\|\sum_{n=0}^{\infty} \sum_{j=1}^{2^{n}} \frac{d_{j}^{(n)}}{j-1+2^{n}} e_{2^{n}+j-1}\right\|_{\widetilde{\ell}_{1}} \\
& \leq\left\|\sum_{n=0}^{\infty} \max _{j=1, \ldots, 2^{n}} d_{j}^{(n)} \cdot 2^{-n} e_{2^{n+1}}\right\|_{\widetilde{\ell}_{1}} \leq 2 \sum_{n=0}^{\infty} \max _{j=1, \ldots, 2^{n}} d_{j}^{(n)}=2\|T c\|_{\left(\oplus_{n=0}^{\infty} \ell_{\infty}^{2^{n}}\right) \ell_{\ell_{1}}} .
\end{aligned}
$$

On the other hand, by the definition of the norm in $\widetilde{\ell}_{1}$ and Theorem 3.3, we have

$$
\begin{aligned}
\|c\|_{\widetilde{\ell}_{1}} & =\left\|\sum_{n=0}^{\infty} \sum_{j=1}^{2^{n}} \frac{d_{j}^{(n)}}{j-1+2^{n}} e_{2^{n}+j-1}\right\|_{\widetilde{\ell}_{1}} \geq \frac{1}{2}\left\|\sum_{n=0}^{\infty} \max _{j=1, \ldots, 2^{n}} d_{j}^{(n)} \cdot 2^{-n} e_{2^{n}}\right\|_{{\widetilde{\ell_{1}}}_{1}} \\
& \geq \frac{1}{72} \sum_{n=0}^{\infty} \max _{j=1, \ldots, 2^{n}} d_{j}^{(n)}=\frac{1}{72}\|T c\|_{\left(\oplus_{n=0}^{\infty} l_{\infty}^{2^{n}}\right)_{l_{1}},}
\end{aligned}
$$

and therefore $T$ is an isomorphism from $\widetilde{\ell_{1}}$ onto $\left(\oplus_{n=0}^{\infty} \ell_{\infty}^{2^{n}}\right)_{\ell_{1}}$. Since $\left(\widetilde{\ell_{1}}\right)^{\prime}=\operatorname{ces}_{\infty}$, by duality, we deduce that $\operatorname{ces}_{\infty}$ is isomorphic to the space $\left(\oplus_{n=0}^{\infty} \ell_{1}^{2^{n}}\right)_{\ell_{\infty}}$.

To get the last result of the corollary, we note that $\left(\oplus_{n=0}^{\infty} \ell_{\infty}^{n}\right) \ell_{1}$ is a complemented subspace of $\left(\oplus_{n=0}^{\infty} \ell_{\infty}^{2^{n}}\right)_{\ell_{1}}$ and hence of $\widetilde{l_{1}}$. Thus, applying the Hagler-Stegall theorem [HS73, Theorem 1], we conclude that the dual space, i.e., $\operatorname{ces}_{\infty}$, contains a complemented subspace isomorphic to $L_{1}[0,1]$.

Remark 1 Bourgain [B81, p. 19] proved that an arbitrary $\ell_{1}$-sum of finite-dimensional Banach spaces has the Schur property (see also [CI90, pp. 60-61] for a simpler proof). Hence, from Corollary 3.5 we can infer that $\widetilde{\ell}_{1}$ has the Schur property and thereby we get another proof of Theorem 3.1.

From Corollary 3.5 and the fact that $\ell_{\infty}$ is a prime space [AK06, Theorem 5.6.5] and [LT77, Theorem 2.a.7]) we have the following.

Corollary 3.6 The spaces $\operatorname{ces}_{\infty}$ and $\ell_{\infty}$ are not isomorphic.

The fact that the space ces $\infty$ contains a complemented subspace isomorphic to $L_{1}[0,1]$ will be a crucial tool in proving the existence of an isomorphism between 
$\mathrm{Ces}_{\infty}$ spaces of functions and sequences. So, it is worth giving its direct proof without referring to the general theorem of Hagler-Stegall [HS73, Theorem 1], especially, because the following proof is interesting on its own.

Theorem 3.7 The space ces $_{\infty}$ contains a complemented subspace isomorphic to

$$
L_{1}[0,1] \text {. }
$$

Proof Thanks to Corollary 3.5, it is sufficient to prove that in the space $\left(\oplus_{n=0}^{\infty} \ell_{1}^{2^{n}}\right) \ell_{\infty}$ there is a complemented subspace isomorphic to $L_{1}[0,1]$. Denote the collection of dyadic intervals of $[0,1]$ by $B_{k}^{n}=\left[\frac{k-1}{2^{n}}, \frac{k}{2^{n}}\right)$, where $k=1, \ldots, 2^{n}$ and $n=0,1,2, \cdots$ and define a sequence of operators $H_{n}: L^{1} \rightarrow \ell_{1}^{2^{n}}$ by

$$
H_{n}: f \mapsto\left\{\int_{B_{n}^{k}} f(t) d t\right\}_{k=1}^{2^{n}} .
$$

Then $\left\|H_{n}\right\|=1$ for each $n$. Moreover, put $H: f \mapsto \oplus_{n=0}^{\infty} H_{n} f$. Then $H$ maps $L_{1}[0,1]$ into the space $\left(\oplus_{n=0}^{\infty} \ell_{1}^{2^{n}}\right) \ell_{\infty}$ with $\|H\|=1$. Moreover, let us show that $H$ is an isometry between the spaces $L_{1}[0,1]$ and $H\left(L_{1}[0,1]\right)$. In fact, denoting by $\left\{h_{k}\right\}$, the Haar basis, for a given $f \in L_{1}[0,1]$ and any $\varepsilon>0$, one can find a function $g=\sum_{k=1}^{N} a_{k} h_{k}$ such that $\|g-f\| \leq \varepsilon$. Then, for $n$ large enough, there holds $\left\|H_{n} g\right\|=\|g\|$, which, in consequence, gives $\left\|H_{n} f\right\| \geq\left\|H_{n} g\right\|-\varepsilon \geq\|f\|-2 \varepsilon$ and proves our claim.

To see that $H\left(L_{1}[0,1]\right)$ is complemented in $\left(\oplus_{n=0}^{\infty} \ell_{1}^{2^{n}}\right)_{\ell_{\infty}}$ a little more work is required. For a given $n$ we set $T_{n}:\left(\oplus_{n=0}^{\infty} \ell_{1}^{2^{n}}\right) \ell_{\infty} \rightarrow L_{1}[0,1]$ by

$$
T_{n}: x \mapsto \sum_{k=1}^{2^{n}} 2^{n} x_{n}^{k} \chi_{B_{n}^{k}}
$$

where $x_{n}=\left(x_{n}^{k}\right)_{k=1}^{2^{n}}$ and $x=\bigoplus_{n=0}^{\infty} x_{n}$. Of course, $\left\|T_{n}\right\|=1$ for each $n$. Let $\eta$ be a free ultrafilter. Then for a given $x \in\left(\oplus_{n=0}^{\infty} \ell_{1}^{2^{n}}\right) \ell_{\infty}$ we define the functional $R_{x}$ on $C[0,1]$ by the formula $R_{x}(g)=\lim _{\eta}\left\langle T_{n} x, g\right\rangle$ for $g \in C[0,1]$. Since $\left\|T_{n}\right\|=1, n \in \mathbb{N}$, we get $\left\|R_{x}\right\|=1$.

Recalling that the space $C[0,1]^{*}$ consists of all regular Borel measures on $[0,1]$ with finite variation, denote by $Q$ the Lebesgue projection that maps any such measure into its absolutely continuous part. Now one can verify that $P: x \mapsto H\left(Q\left(R_{x}\right)\right)$ is the required projection from $\left(\oplus_{n=0}^{\infty} \ell_{1}^{2^{n}}\right) \ell_{\infty}$ onto $H\left(L_{1}[0,1]\right)$. In fact, since $\left\{T_{n}(H f)\right\}_{n=0}^{\infty}$ is a uniformly integrable martingale associated with a function $f \in L_{1}[0,1]$, there holds $T_{n}(H f) \rightarrow f$ in $L_{1}[0,1]$-norm. In consequence,

$$
R_{H f}(g)=\lim _{\eta}\left\langle T_{n} H f, g\right\rangle=\lim _{n \rightarrow \infty}\left\langle T_{n} H f, g\right\rangle=\langle f, g\rangle \quad \text { for } g \in C[0,1] .
$$

Therefore, $Q\left(R_{H f}\right)=f$, which proves our claim.

The second named author learned yet another original proof of the above theorem from Professor Yves Raynaud. Namely, it is possible to construct a projection from $\left(\oplus_{n=0}^{\infty} \ell_{1}^{2^{n}}\right)_{\ell_{\infty}}$ onto the space, say $M$, of all bounded dyadic martingales in $L_{1}$ and then, in the second step, from $M$ onto $L_{1}$.

Now we investigate the conditions under which Cesàro and Tandori sequence spaces have or do not have the Dunford-Pettis property. 
Observe that under the assumption of nontriviality of indices of a function $\varphi$, that is, when $0<p_{\varphi} \leq q_{\varphi}<1$, in the case when $I=[0, \infty)$, we have $C \Lambda_{\varphi}=L_{1}(\varphi(t) / t)$ with equivalent norms [DS07, Theorem 4.4] and [LM15a, Theorem 8]). And hence, by Theorem 2.1 (i), the corresponding Tandori space $\widetilde{M_{\varphi}}=L_{\infty}(\varphi)$, so they both have the Dunford-Pettis property. An inspection of the proof of [LM15a, Theorem 8] combined with duality (see Theorem 2.1 (iii)) shows that for the respective sequence spaces the following result holds.

Theorem 3.8 Let $\varphi$ be an increasing concave function on $[0, \infty)$.

(i) If $p_{\varphi}^{\infty}>0$, then $C \lambda_{\varphi}=\ell_{1}(\varphi(n) / n)$ and therefore has the Dunford-Pettis property.

(ii) If $q_{\varphi}^{\infty}<1$, then $\widetilde{m_{\varphi}}=\ell_{\infty}(\varphi(n))$ and therefore has the Dunford-Pettis property.

In the proof of a similar result related to the spaces $\widetilde{\lambda_{\varphi}}$ and $C m_{\varphi}$ we will make use of a suitable isomorphic description of these spaces and the well-known results of Bourgain mentioned in the introduction [Bo81].

Theorem 3.9 For an arbitrary increasing concave function $\varphi$ on $[0, \infty)$ the spaces $\widetilde{\lambda_{\varphi}}$ and $\mathrm{Cm}_{\varphi}$ have the Dunford-Pettis property.

Proof At first, in the case when $\lim _{t \rightarrow \infty} \varphi(t)<\infty$ we have $\lambda_{\varphi}=\ell_{\infty}$, whence $\widetilde{\lambda_{\varphi}}=$ $\ell_{\infty}$, and the result follows. So, let $\lim _{t \rightarrow \infty} \varphi(t)=\infty$. Moreover, the function $\varphi(t)$ is strictly increasing and, without loss of generality, we can assume that $\varphi(1)=1$. Let us define the increasing sequence $\left\{n_{k}\right\}_{k=1}^{\infty}$, where $n_{1}=1$, as follows

$$
n_{k+1}:=\sup \left\{i>n_{k}: \varphi(i)-\varphi\left(n_{k}\right) \leq 2^{k}\right\}, \quad k=1,2, \ldots
$$

Then, since $\varphi\left(n_{k+1}+1\right)-\varphi\left(n_{k}\right)>2^{k}$ and, by subadditivity of $\varphi$,

$$
\varphi\left(n_{k+1}+1\right)-\varphi\left(n_{k+1}\right) \leq \varphi(1)=1,
$$

we have

$$
2^{k-1} \leq \varphi\left(n_{k+1}\right)-\varphi\left(n_{k}\right) \leq 2^{k}, \quad k=1,2, \ldots
$$

Therefore, by (3.3) and [GHS96, Proposition 2.1] (see also [GP03, Lemma 3.2]), for every $x=\left(x_{n}\right)_{n=1}^{\infty} \in \widetilde{\lambda_{\varphi}}$ we have

$$
\begin{aligned}
\|x\|_{\widetilde{\lambda_{\varphi}}} & =\sum_{n=1}^{\infty} \widetilde{x_{n}}(\varphi(n+1)-\varphi(n))=\sum_{k=1}^{\infty} \sum_{n=n_{k}}^{n_{k+1}-1} \widetilde{x_{n}}(\varphi(n+1)-\varphi(n)) \\
& \leq \sum_{k=1}^{\infty} \widetilde{x_{n_{k}}}\left(\varphi\left(n_{k+1}\right)-\varphi\left(n_{k}\right)\right) \leq \sum_{k=1}^{\infty} 2^{k} \sup _{j \geq k} \max _{n_{j} \leq i \leq n_{j+1}}\left|x_{i}\right| \\
& \leq \sum_{k=1}^{\infty} 2^{k} \sum_{j=k}^{\infty} \max _{n_{j} \leq i \leq n_{j+1}}\left|x_{i}\right|=\sum_{j=1}^{\infty} \max _{n_{j} \leq i \leq n_{j+1}}\left|x_{i}\right| \sum_{k=1}^{j} 2^{k} \\
& \leq 2 \sum_{j=1}^{\infty} 2^{j} \max _{n_{j} \leq i \leq n_{j+1}}\left|x_{i}\right|=2\|x\|_{\oplus},
\end{aligned}
$$

where $\|x\|_{\oplus}:=\sum_{j=1}^{\infty} 2^{j} \max _{n_{j} \leq i \leq n_{j+1}}\left|x_{i}\right|$. 
Conversely, again, by (3.3),

$$
\begin{aligned}
\|x\|_{\oplus} & =2 \max _{1 \leq i \leq n_{2}}\left|x_{i}\right|+\sum_{j=2}^{\infty} 2^{j} \max _{n_{j} \leq i \leq n_{j+1}}\left|x_{i}\right| \leq 2\|x\|_{\ell_{\infty}}+4 \sum_{j=2}^{\infty} \widetilde{x_{n_{j}}}\left[\varphi\left(n_{j}\right)-\varphi\left(n_{j-1}\right)\right] \\
& \leq 2\|x\|_{\ell_{\infty}}+4 \sum_{j=2}^{\infty} \sum_{n=n_{j-1}}^{n_{j}-1} \widetilde{x_{n}}[\varphi(n+1)-\varphi(n)] \leq\left(\frac{2}{\varphi(2)-1}+4\right)\|x\|_{\widetilde{\lambda_{\varphi}}} .
\end{aligned}
$$

These inequalities show that $\widetilde{\lambda_{\varphi}}$ is isomorphic to the space $\left(\oplus_{k \in \mathbb{N}} \ell_{\infty}^{m_{k}}\right)_{\ell_{1}}$, where $m_{k}=$ $n_{k+1}-n_{k}, k \in \mathbb{N}$. Hence, applying [Bo81, Corollary7], we obtain that the space $\widetilde{\lambda_{\varphi}}$ has the Dunford-Pettis property.

Regarding $\mathrm{Cm}_{\varphi}$ we first note that in the case when $\lim _{t \rightarrow \infty} \varphi(t) / t>0$, the latter space coincides with $\ell_{1}$ and hence has the Dunford-Pettis property. If

$$
\lim _{t \rightarrow \infty} \varphi(t) / t=0 \text {, }
$$

then from Theorem 2.1 (iii) and the first part of the proof it follows that

$$
\left(C m_{\varphi}\right)^{\prime}=\widetilde{\lambda_{\psi}} \simeq\left(\bigoplus_{k \in \mathbb{N}} \ell_{\infty}^{m_{k}}\right) \ell_{1},
$$

where $\psi(t)=t / \varphi(t)$. Combining this with the fact that $\mathrm{Cm}_{\varphi}$ has the Fatou property, we infer $C m_{\varphi} \simeq\left[\left(\oplus_{k \in \mathbb{N}} \ell_{\infty}^{m_{k}}\right) \ell_{1}\right]^{\prime}=\left(\oplus_{k \in \mathbb{N}} \ell_{1}^{m_{k}}\right)_{\ell_{\infty}}$, Hence, from Bourgain's result [Bo81, Theorem 1] it follows that $\mathrm{Cm}_{\varphi}$ has the Dunford-Pettis property.

Corollary 3.10 For any increasing concave function $\varphi$ on $[0, \infty)$ the space $C\left(m_{\varphi}^{0}\right)$ has the Dunford-Pettis property.

Proof Since the space $C\left(m_{\varphi}^{0}\right)$ is order continuous, by Theorem 2.1 (iii), we have

$$
\left[C\left(m_{\varphi}^{0}\right)\right]^{*}=\left[C\left(m_{\varphi}^{0}\right)\right]^{\prime}=\widetilde{\lambda_{\psi}},
$$

where $\psi(t)=t / \varphi(t)$. Now the desired result follows from the preceding theorem.

Now we show that in the case of reflexive spaces the situation is completely different.

Theorem 3.11 Let $X$ be a reflexive symmetric sequence space.

(i) If the operator $C_{d}$ is bounded on $X$, then $C X$ does not have the Dunford-Pettis property.

(ii) If the operator $C_{d}$ is bounded on $X^{\prime}$, then $\widetilde{X}$ does not have the Dunford-Pettis property.

Proof (i) Since $X$ is reflexive, it follows that $C_{d}: C X \rightarrow X$ is a weakly compact operator. Therefore, it is sufficient to show that $C_{d}$ is not a Dunford-Pettis operator.

Consider the sequence $x_{n}=\varphi_{X^{\prime}}(n) e_{n}$, where $\left\{e_{n}\right\}$ is the canonical basis in $X$ and $\varphi_{X^{\prime}}$ is the fundamental function of $X^{\prime}$. Let us show that $x_{n} \rightarrow 0$ weakly in $C X$.

Since the space $X$ has an order continuous norm, $C X$ also has order continuous norm and, by Theorem 2.1 (iii), we obtain $(C X)^{*}=(C X)^{\prime}=\widetilde{X}^{\prime}$. Therefore, by the definition of $\widetilde{X}^{\prime}$, it is sufficient to prove that

$$
\left\langle y, x_{n}\right\rangle=\varphi_{X^{\prime}}(n) y_{n} \rightarrow 0 \text { as } n \rightarrow \infty
$$


for each non-increasing positive sequence $y=\left(y_{n}\right) \in X^{\prime}$. Observe that $X^{\prime} \subset m_{\varphi_{X^{\prime}}}$, where $m_{\varphi_{X^{\prime}}}$ is the Marcinkiewicz space with the fundamental function $\varphi_{X^{\prime}}$. Moreover, by reflexivity of $X, X^{\prime}=\left(X^{\prime}\right)^{0}$, and thus

$$
X^{\prime} \subset m_{\varphi_{X^{\prime}}}^{0}=\left\{\left(z_{n}\right): \lim _{n \rightarrow \infty} \varphi_{X^{\prime}}(n) z_{n}^{*}=0\right\} .
$$

Clearly, this embedding implies (3.4) and hence $x_{n} \rightarrow 0$ weakly in $C X$.

On the other hand, $C_{d} e_{n}=\sum_{k=n}^{\infty} e_{k} / k$ for every $n \in \mathbb{N}$. Since $X$ is a symmetric space, we have

$$
\begin{aligned}
\left\|C_{d} e_{n}\right\|_{X} & =\left\|\sum_{k=1}^{\infty} \frac{e_{k}}{n+k-1}\right\|_{X} \geq\left\|\sum_{k=1}^{n} \frac{e_{k}}{n+k-1}\right\|_{X} \\
& \geq \frac{1}{2 n}\left\|\sum_{k=1}^{n} e_{k}\right\|_{X}=\frac{\varphi_{X}(n)}{2 n}=\frac{1}{2 \varphi_{X^{\prime}}(n)} .
\end{aligned}
$$

Hence, $\left\|C_{d} x_{n}\right\|_{X} \geq 1 / 2$ for all $n$, and the proof is completed

(ii) Since $X^{\prime}$ is reflexive and $C_{d}$ is bounded on $X^{\prime}$, from Theorem 3.11 (i) it follows that $C\left(X^{\prime}\right)$ does not have the Dunford-Pettis property. By duality, Theorem 2.1 (iii) and the fact that $C\left(X^{\prime}\right)$ is order continuous, we obtain $\widetilde{X}=\left(C X^{\prime}\right)^{\prime}=\left(C X^{\prime}\right)^{*}$. Thus, $\widetilde{X}$ is the dual space to a space without the Dunford-Pettis property. So, $\widetilde{X}$ also fails to have it.

\section{On the Dunford-Pettis Property of Cesàro and Tandori Function Spaces}

As mentioned above, under the assumption of nontriviality of indices of a function $\varphi$, the spaces $C \Lambda_{\varphi}$ and $\widetilde{M_{\varphi}}$ are some weighted $L_{1}$-spaces and $L_{\infty}$-spaces, respectively, and so they both have the Dunford-Pettis property (see [DS07, Theorem 4.4], [LM15a, Theorem 8]). Similarly, as in Theorem 3.8, we are able to prove the latter property also for their counterparts, $\widetilde{\Lambda_{\varphi}}$ and $C M_{\varphi}$.

Theorem 4.1 Let $\varphi$ be an increasing concave function on $[0, \infty)$.

(i) The space $\widetilde{\Lambda_{\varphi}}[0, \infty)$ is isomorphic to the space $\left(\oplus_{n \in \mathbb{N}} L_{\infty}[0,1]\right)_{\ell_{1}}$ and has the Dunford-Pettis property.

(ii) If $q_{\varphi}<1$, then the space $C M_{\varphi}[0, \infty)$ is isomorphic to the space $\left(\oplus_{n \in \mathbb{N}} L_{1}[0,1]\right)_{\ell_{\infty}}$ and has the Dunford-Pettis property.

First, we prove the following auxiliary result.

Proposition 4.2 Let $w$ be a locally integrable function on $[0, \infty), w(t)>0$ almost everywhere, such that $\int_{0}^{\infty} w(t) d t=\infty$. For $1 \leq p<\infty$, we consider the weighted $L_{p}$-space with the norm $\|f\|_{L_{p}(w)}:=\left(\int_{0}^{\infty}|f(t)|^{p} w(t) d t\right)^{1 / p}$. Then the space $\overline{L_{p}(w)}$ is isomorphic to the space $\left(\oplus_{n \in \mathbb{Z}} L_{\infty}[0,1]\right)_{\ell_{p}}$, and the constant of isomorphism depends only on $w$.

Proof Without loss of generality, we can assume that $\int_{0}^{1} w(t) d t=\frac{1}{a-1}$, where $a>1$. Thanks to the assumptions, there is an increasing sequence $\left\{t_{k}\right\}_{k \in \mathbb{Z}}$ such that $t_{0}=$ 
$1, t_{n} \rightarrow 0$ as $n \rightarrow-\infty, t_{n} \rightarrow+\infty$ as $n \rightarrow+\infty$ and

$$
\int_{t_{n}}^{t_{n+1}} w(t) d t=a^{n} \quad \text { for all } n \in \mathbb{Z} .
$$

Then, applying once more [GHS96, Proposition 2.1] (see also [GP03, Lemma 3.2]), for every $f \in \overline{L_{p}(w)}$ we have

$$
\begin{aligned}
\|f\| \frac{p}{L_{p}(w)} & =\int_{0}^{\infty} \widetilde{f(t)} p(t) d t=\sum_{n \in \mathbb{Z}} \int_{t_{n}}^{t_{n+1}} \operatorname{ess} \sup _{s \geq t}|f(s)|^{p} w(t) d t \\
& \leq \sum_{n \in \mathbb{Z}} \operatorname{ess} \sup _{s \geq t_{n}}|f(s)|^{p} a^{n}=\sum_{n \in \mathbb{Z}} \sup _{k \geq n} \operatorname{ess} \sup _{t_{k} \leq s \leq t_{k+1}}|f(s)|^{p} a^{n} \\
& \leq C(a) \sum_{n \in \mathbb{Z}}\left\|f \chi_{\left[t_{n}, t_{n+1}\right]}\right\|_{L_{\infty}}^{p} a^{n}=C(a)\|f\|_{\oplus}^{p},
\end{aligned}
$$

where

$$
\|f\|_{\oplus}:=\left(\sum_{n \in \mathbb{Z}} a^{n}\left\|f \chi_{\left[t_{n}, t_{n+1}\right]}\right\|_{L_{\infty}}^{p}\right)^{1 / p}
$$

and $C(a)$ is some constant depending only on $a$ (and hence on $w$ ). On the other hand,

$$
\begin{aligned}
\|f\|_{\oplus}^{p} & =a \sum_{n \in \mathbb{Z}} a^{n-1}\left\|f \chi_{\left[t_{n}, t_{n+1}\right]}\right\|_{L_{\infty}}^{p}=a \sum_{n \in \mathbb{Z}} \int_{t_{n-1}}^{t_{n}} \operatorname{ess~sup~}_{t_{n} \leq s \leq t_{n+1}}|f(s)|^{p} w(t) d t \\
& \leq a \sum_{n \in \mathbb{Z}} \int_{t_{n-1}}^{t_{n}} \operatorname{ess} \sup |f(s)|^{p} w(t) d t=a\|f\| \frac{p}{L_{p}(w)} .
\end{aligned}
$$

Since the space $L_{\infty}[a, b]$ is isomorphic to the space $L_{\infty}[0,1]$ for every $0<a<b<\infty$, the result follows.

Proof of Theorem 4.1 (i) It is clear that $\widetilde{\Lambda_{\varphi}}=\overline{L_{1}\left(\varphi^{\prime}\right)}$. Therefore, from Proposition 4.2 it follows that $\widetilde{\Lambda_{\varphi}} \simeq\left(\bigoplus_{n \in \mathbb{N}} L_{\infty}[0,1]\right)_{\ell_{1}}$ and, by Bourgain's result [Bo81, Corollary 7], $\widetilde{\Lambda_{\varphi}}$ has the Dunford-Pettis property.

(ii) Since $q_{\varphi}<1$, then the operator $C$ is bounded in $M_{\varphi}[0, \infty)$ [KPS82, Theorem 6.6, p. 138] and hence, by Theorem 2.1 (i), the Köthe dual of the space $C M_{\varphi}$ coincides with the space $\widetilde{\Lambda_{\psi}}$, where $\psi(t)=t / \varphi(t), t>0$. Therefore, applying Proposition 4.2, we are able to get the result, arguing in the same way as in the concluding part of the proof of Theorem 3.8.

Theorem 4.3 The spaces $\operatorname{Ces}_{\infty}(I)$ and $\widetilde{L_{1}}(I)$, where $I=[0, \infty)$ or $[0,1]$, have the Dunford-Pettis property and they are not isomorphic.

Proof At first, let $I=[0, \infty)$. Since $\widetilde{L_{1}}=\widetilde{\Lambda_{\varphi_{1}}}$, where $\varphi_{1}(t)=t$, and $\operatorname{Ces}_{\infty}=C L_{\infty}=$ $C M_{\varphi_{0}}$, where $\varphi_{0}(t)=1$, by Theorem 4.1 , the spaces $\widetilde{L_{1}}$ and $\operatorname{Ces}_{\infty}$ have the DunfordPettis property.

Let us show that $\widetilde{L_{1}}$ and $\operatorname{Ces}_{\infty}$ are not isomorphic. By Theorem 4.1, the space $\widetilde{L_{1}}$ is isomorphic to the space $\left(\bigoplus_{n \in \mathbb{N}} L_{\infty}[0,1]\right)_{\ell_{1}}$ and therefore, according to Pełczyński's result on isomorphism between $L_{\infty}$ and $\ell_{\infty}$ ([Pe58]; see also [AK06, Theorem 4.3.10]), $\widetilde{L_{1}}$ is isomorphic also to $\left(\bigoplus_{n \in \mathbb{N}} \ell_{\infty}\right) \ell_{1}$. Since the latter space fails to contain a complemented subspace isomorphic to $L_{1}[0,1]$ [CM08, Proposition 3], then $\widetilde{L_{1}}$ also does 
not. On the other hand, the space $\mathrm{Ces}_{\infty}$ contains such a complemented subspace (see Proposition 2.2), and the result follows.

It is easy to see that the assertion of Proposition 4.2 holds also for weighted $L_{p}$-spaces on $[0,1]$ (with the same proof). Therefore, $\widetilde{L_{1}}[0,1] \simeq\left(\oplus_{n \in \mathbb{N}} L_{\infty}[0,1]\right)_{\ell_{1}}$ and, since $\left(\operatorname{Ces}_{\infty}[0,1]\right)^{\prime}=\widetilde{L_{1}}[0,1]$, then $\operatorname{Ces}_{\infty}[0,1] \simeq\left(\oplus_{n \in \mathbb{N}} L_{1}[0,1]\right)_{\ell_{\infty}}$. Thus, the result can be proved in the same way as in the case of $[0, \infty)$.

A similar result can also be deduced from Theorem 4.1 for the separable part of a Marcinkiewicz space $M_{\varphi}^{0}$ (see also Corollary 3.10). In fact, since the space $C\left(M_{\varphi}^{0}\right)$ on $[0, \infty)$ is order continuous and the condition $\beta_{M_{\varphi}^{0}}=q_{\varphi}<1$ implies the boundedness of the operator $C$ on $M_{\varphi}^{0}$, by Theorem $2.1(\mathrm{i})$, we have $\left[C\left(M_{\varphi}^{0}\right)\right]^{*}=\left[C\left(M_{\varphi}^{0}\right)\right]^{\prime}=\widetilde{\Lambda_{\psi}}$. As a result, applying Theorem $4.1(\mathrm{i})$, we get that $C\left(M_{\varphi}^{0}\right)$ has the Dunford-Pettis property. However, we prefer to give the more direct proof of the latter fact (without exploiting Bourgain's results [Bo81]) by using the following property of separable Cesáro-Marcinkiewicz spaces.

Proposition 4.4 Suppose that $\varphi$ is an increasing concave function on $[0, \infty)$ such that $\lim _{t \rightarrow 0^{+}} \varphi(t)=0$ and $q_{\varphi}<1$. Let $X=C\left(M_{\varphi}^{0}\right)$ on $[0, \infty)$ and let $I_{n}:=\left[a_{n}, b_{n}\right]$ be a sequence of intervals from $[0, \infty)$ such that either

$$
b_{1}>a_{1}>b_{2}>a_{2}>\cdots>0 \text { and } b_{n} \rightarrow 0^{+} \text {as } n \rightarrow \infty
$$

or

$$
a_{1}<b_{1}<a_{2}<b_{2}<\cdots \text { and } a_{n} \rightarrow \infty \text { as } n \rightarrow \infty \text {. }
$$

Then there are a subsequence of positive integers $\left\{n_{k}\right\}_{k=1}^{\infty}, n_{1}<n_{2}<\cdots$ and a constant $C>0$ such that for every sequence $\left\{x_{n}\right\} \subset X$ satisfying the condition supp $x_{n} \subset I_{n}, n=$ $1,2, \ldots$, we have

$$
\max _{k=1, \ldots, m}\left\|x_{n_{k}}\right\|_{X} \leq\left\|\sum_{k=1}^{m} x_{n_{k}}\right\|_{X} \leq C \max _{k=1, \ldots, m}\left\|x_{n_{k}}\right\|_{X}, m=1,2, \ldots
$$

Proof Since a given sequence $\left\{x_{n}\right\}$ under consideration consists of pairwise disjoint functions, the left inequality in (4.3) holds for an arbitrary subsequence $\left\{n_{k}\right\}_{k=1}^{\infty}$. So, we need only to prove the reverse inequality. Obviously, we may assume that $x_{n} \geq 0$ almost everywhere. Since $q_{\varphi}<1$, then $\lim _{t \rightarrow 0^{+}} \frac{t}{\varphi(t)}=0$. Therefore, in the case (4.1), applying the diagonal procedure, from any given sequence $\left\{I_{n}\right\}$ we can extract a subsequence of intervals (which we will denote still by $I_{n}=\left[a_{n}, b_{n}\right]$ ) such that

$$
\sum_{k=n+1}^{\infty} \psi\left(b_{k}\right) \leq \psi\left(a_{n}\right), \quad \text { where } \psi(t)=t / \varphi(t)
$$

We claim that the corresponding sequence of functions (still denoting them by $\left\{x_{n}\right\}$ and assuming that $\operatorname{supp} x_{n} \subset I_{n}$ ) satisfies the right-hand inequality in (4.3). For any 
$m \in \mathbb{N}$ and $t \in(0, \infty)$, we have

$$
\begin{aligned}
\frac{1}{t} \int_{0}^{t}\left(\sum_{k=1}^{m} x_{k}(s)\right) d s=\frac{1}{t} \sum_{j=2}^{m} \sum_{i=m-j+2}^{m} \int_{a_{i}}^{b_{i}} x_{i}(s) d s \chi_{\left[a_{m-j+1}, b_{m-j+1}\right]}(t) \\
+\frac{1}{t} \sum_{j=1}^{m} \int_{a_{m-j+1}}^{t} x_{m-j+1}(s) d s \chi_{\left[a_{m-j+1}, b_{m-j+1}\right]}(t) \\
+\frac{1}{t} \sum_{j=1}^{m} \sum_{i=m-j+1}^{m} \int_{a_{i}}^{b_{i}} x_{i}(s) d s \chi_{\left[b_{m-j+1}, a_{m-j}\right]}(t) \\
=f_{1}(t)+f_{2}(t)+f_{3}(t),
\end{aligned}
$$

where $a_{0}=\infty$, and

$$
\begin{aligned}
& f_{1}(t):=\frac{1}{t} \sum_{j=2}^{m} \sum_{i=m-j+2}^{m} \int_{a_{i}}^{b_{i}} x_{i}(s) d s \chi_{\left[a_{m-j+1}, a_{m-j}\right]}(t), \\
& f_{2}(t):=\frac{1}{t} \sum_{j=1}^{m} \int_{a_{m-j+1}}^{b_{m-j+1}} x_{m-j+1}(s) d s \chi_{\left[b_{m-j+1}, a_{m-j}\right]}(t), \\
& f_{3}(t):=\frac{1}{t} \sum_{j=1}^{m} \int_{a_{m-j+1}}^{t} x_{m-j+1}(s) d s \chi_{\left[a_{m-j+1}, b_{m-j+1}\right]}(t) .
\end{aligned}
$$

Since $\beta_{M_{\varphi}^{0}}=q_{\varphi}<1$, the operator $C$ is bounded in $M_{\varphi}^{0}$ [KPS82, Theorem 6.6], and hence, by Theorem $2.1(\mathrm{i}),(C X)^{*}=(C X)^{\prime}=\widetilde{X^{\prime}}=\widetilde{\Lambda_{\psi}}$ with equivalent norms. Thus, by (2.1) and (2.8), for arbitrary $0<a<b<\infty$ and $x \in X$,

$$
\int_{a}^{b}|x(s)| d s \leq C_{1}\|x\|_{X}\left\|\chi_{[a, b]}\right\|_{\widetilde{\Lambda}_{\psi}}=C_{1}\|x\|_{X} \psi(b) .
$$

Hence, by (4.4),

$$
\begin{aligned}
f_{1}(t) & \leq \frac{C_{1}}{t} \sum_{j=2}^{m} \sum_{i=m-j+2}^{m} \psi\left(b_{i}\right)\left\|x_{i}\right\|_{X} \chi_{\left[a_{m-j+1}, a_{m-j}\right]}(t) \\
& \leq \frac{C_{1}}{t} \sum_{j=2}^{m} \psi\left(a_{m-j+1}\right) \chi_{\left[a_{m-j+1}, a_{m-j}\right]}(t) \cdot \max _{i=1, \ldots, m}\left\|x_{i}\right\|_{X} \\
& \leq \frac{C_{1}}{\varphi(t)} \sum_{j=2}^{m} \chi_{\left[a_{m-j+1}, a_{m-j}\right]}(t) \cdot \max _{i=1, \ldots, m}\left\|x_{i}\right\|_{X} \leq \frac{C_{1}}{\varphi(t)} \max _{i=1, \ldots, m}\left\|x_{i}\right\|_{X},
\end{aligned}
$$

whence, since $q_{\varphi}<1$, by (2.5), it follows that

$$
\left\|f_{1}\right\|_{M_{\varphi}} \leq C_{1} \frac{\varphi(t)}{t} \int_{0}^{t} \frac{1}{\varphi(s)} d s \max _{i=1, \ldots, m}\left\|x_{i}\right\|_{X} \leq C_{1} C_{2} \max _{i=1, \ldots, m}\left\|x_{i}\right\|_{X} .
$$

Similarly, since $\psi$ increases, we have

$$
\begin{aligned}
f_{2}(t) & \leq \frac{C_{1}}{t} \sum_{j=1}^{m} \psi\left(b_{m-j+1}\right)\left\|x_{m-j+1}\right\|_{X} \cdot \chi_{\left[b_{m-j+1}, a_{m-j}\right]}(t) \\
& \leq \frac{C_{1}}{\varphi(t)} \sum_{j=1}^{m} \chi_{\left[b_{m-j+1}, a_{m-j}\right]}(t) \max _{i=1, \ldots, m}\left\|x_{i}\right\|_{X} \leq \frac{C_{1}}{\varphi(t)} \max _{i=1, \ldots, m}\left\|x_{i}\right\|_{X},
\end{aligned}
$$


and again

$$
\left\|f_{2}\right\|_{M_{\varphi}} \leq C_{1} C_{2} \max _{i=1, \ldots, m}\left\|x_{i}\right\|_{X} .
$$

Finally, using (4.5) once more, we have

$$
\begin{aligned}
f_{3}(t) & \leq \frac{C_{1}}{t} \sum_{j=1}^{m} \psi(t)\left\|x_{m-j+1}\right\|_{X} \cdot \chi_{\left[a_{m-j+1}, b_{m-j+1}\right]}(t) \\
& \leq \frac{C_{1}}{\varphi(t)} \sum_{j=1}^{m} \chi_{\left[a_{m-j+1}, b_{m-j+1}\right]}(t) \max _{i=1, \ldots, m}\left\|x_{i}\right\|_{X} \leq \frac{C_{1}}{\varphi(t)} \max _{i=1, \ldots, m}\left\|x_{i}\right\|_{X},
\end{aligned}
$$

whence again

$$
\left\|f_{3}\right\|_{M_{\varphi}} \leq C_{1} C_{2} \max _{i=1, \ldots, m}\left\|x_{i}\right\|_{X} .
$$

Thus, from (4.5)-(4.8) it follows that

$$
\left\|\sum_{k=1}^{m} x_{k}\right\|_{X} \leq\left\|f_{1}\right\|_{M_{\varphi}}+\left\|f_{2}\right\|_{M_{\varphi}}+\left\|f_{3}\right\|_{M_{\varphi}} \leq C \max _{i=1, \ldots, m}\left\|x_{i}\right\|_{X}
$$

where the constant $C:=3 C_{1} C_{2}$ depends only on the function $\varphi$.

Regarding the case (4.2), we note that condition $q_{\varphi}<1 \mathrm{implies} \lim _{t \rightarrow \infty} \frac{t}{\varphi(t)}=\infty$. Hence, from any given sequence of intervals we can select a subsequence of intervals (denoted still by $I_{n}=\left[a_{n}, b_{n}\right]$ ) such that

$$
\sum_{i=1}^{k-1} \psi\left(b_{i}\right) \leq \psi\left(a_{k}\right), k=2,3, \ldots
$$

For arbitrary $m \in \mathbb{N}$ and $t>0$, we have

$$
\begin{aligned}
\frac{1}{t} \int_{0}^{t}\left(\sum_{k=1}^{m} x_{k}(s)\right) d s= & \frac{1}{t} \sum_{k=1}^{m}\left(\sum_{i=1}^{k-1} \int_{a_{i}}^{b_{i}} x_{i}(s) d s+\int_{a_{k}}^{t} x_{k}(s) d s\right) \chi_{\left[a_{k}, b_{k}\right]}(t) \\
& +\frac{1}{t} \sum_{k=1}^{m} \sum_{i=1}^{k} \int_{a_{i}}^{b_{i}} x_{i}(s) d s \cdot \chi_{\left[b_{k}, a_{k+1}\right]}(t) \\
= & g_{1}(t)+g_{2}(t)+g_{3}(t),
\end{aligned}
$$

where $a_{m+1}=\infty$, and

$$
\begin{aligned}
& g_{1}(t):=\frac{1}{t} \sum_{k=2}^{m} \sum_{i=1}^{k-1} \int_{a_{i}}^{b_{i}} x_{i}(s) d s \cdot \chi_{\left[a_{k}, a_{k+1}\right]}(t) \\
& g_{2}(t):=\frac{1}{t} \sum_{k=1}^{m} \int_{a_{k}}^{b_{k}} x_{i}(s) d s \cdot \chi_{\left[b_{k}, a_{k+1}\right]}(t) \\
& g_{3}(t):=\sum_{k=1}^{m} \int_{a_{k}}^{t} x_{k}(s) d s \cdot \chi_{\left[a_{k}, b_{k}\right]}(t)
\end{aligned}
$$


First, applying (4.5) and (4.9) we obtain

$$
\begin{aligned}
g_{1}(t) & \leq \frac{C_{1}}{t} \sum_{k=2}^{m} \sum_{i=1}^{k-1} \psi\left(b_{i}\right)\left\|x_{i}\right\|_{X} \cdot \chi_{\left[a_{k}, a_{k+1}\right]}(t) \\
& \leq \frac{C_{1}}{t} \sum_{k=2}^{m} \psi\left(a_{k}\right) \cdot \chi_{\left[a_{k}, a_{k+1}\right]}(t) \cdot \max _{i=1, \ldots, m}\left\|x_{i}\right\|_{X} \\
& \leq \frac{C_{1}}{\varphi(t)} \sum_{k=2}^{m} \chi_{\left[a_{k}, a_{k+1}\right]}(t) \cdot \max _{i=1, \ldots, m}\left\|x_{i}\right\|_{X} \leq \frac{C_{1}}{\varphi(t)} \max _{i=1, \ldots, m}\left\|x_{i}\right\|_{X} .
\end{aligned}
$$

Next, similarly,

$$
\begin{aligned}
& g_{2}(t) \leq \frac{C_{1}}{t} \sum_{k=1}^{m} \psi\left(b_{k}\right)\left\|x_{k}\right\|_{X} \cdot \chi_{\left[b_{k}, a_{k+1}\right]}(t) \leq \frac{C_{1}}{\varphi(t)} \max _{i=1, \ldots, m}\left\|x_{i}\right\|_{X}, \\
& g_{3}(t) \leq \frac{C_{1}}{t} \sum_{k=1}^{m} \psi(t)\left\|x_{k}\right\|_{X} \cdot \chi_{\left[a_{k}, b_{k}\right]}(t) \leq \frac{C_{1}}{\varphi(t)} \max _{i=1, \ldots, m}\left\|x_{i}\right\|_{X} .
\end{aligned}
$$

As a result, using (2.5), we have

$$
\left\|\sum_{k=1}^{m} x_{k}\right\|_{X} \leq\left\|g_{1}\right\|_{M_{\varphi}}+\left\|g_{2}\right\|_{M_{\varphi}}+\left\|g_{3}\right\|_{M_{\varphi}} \leq C \max _{i=1, \ldots, m}\left\|x_{i}\right\|_{X}
$$

where the constant $C:=3 C_{1} C_{2}$ depends only on the function $\varphi$.

Corollary 4.5 Let $\varphi$ satisfy all the conditions of Proposition 4.4 and let $X=C\left(M_{\varphi}^{0}\right)$ on $[0, \infty)$. Suppose that $I_{n}:=\left[a_{n}, b_{n}\right], n=1,2, \ldots$, be a sequence of intervals from $[0, \infty)$ such that either $b_{1}>a_{1}>b_{2}>a_{2}>\cdots>0$ and $b_{n} \rightarrow 0^{+}$as $n \rightarrow \infty$ or $a_{1}<b_{1}<a_{2}<b_{2}<\cdots$ and $a_{n} \rightarrow \infty$ as $n \rightarrow \infty$. Then every semi-normalized sequence $\left\{f_{n}\right\} \subset X$ such that supp $f_{n} \subset I_{n}, n=1,2, \ldots$ contains a subsequence $\left\{f_{n_{k}}\right\}$ that is equivalent in $X$ to the canonical basis in $c_{0}$.

Proof At first, applying Proposition 4.4, we find a subsequence of positive integers $\left\{n_{k}\right\}_{k=1}^{\infty}, n_{1}<n_{2}<\cdots$ and a constant $C>0$ such that for every sequence $\left\{x_{n}\right\} \subset X$ with supp $x_{n} \subset I_{n}, n=1,2, \ldots$, we have

$$
\max _{k=1, \ldots, m}\left\|x_{n_{k}}\right\|_{X} \leq\left\|\sum_{k=1}^{m} x_{n_{k}}\right\|_{X} \leq C \max _{k=1, \ldots, m}\left\|x_{n_{k}}\right\|_{X}, \quad m=1,2, \ldots
$$

In particular, setting $x_{n}=c_{k} f_{n}$ if $n_{k} \leq n<n_{k+1}, k=1,2, \ldots$, where $\left(c_{k}\right)$ is an arbitrary sequence from $c_{0}$, and assuming that $D^{-1} \leq\left\|f_{n}\right\|_{X} \leq D, n=1,2, \ldots$ for all $m \in \mathbb{N}$, we obtain

$$
D^{-1} \max _{k=1, \ldots, m}\left|c_{k}\right| \leq\left\|\sum_{k=1}^{m} c_{k} f_{n_{k}}\right\|_{X} \leq C D \max _{k=1, \ldots, m}\left|c_{k}\right| .
$$

Since $\left(c_{k}\right) \in c_{0}$, then the series $\sum_{k=1}^{\infty} c_{k} f_{n_{k}}$ converges in $X$ and we have

$$
D^{-1}\left\|\left(c_{k}\right)\right\|_{c_{0}} \leq\left\|\sum_{k=1}^{\infty} c_{k} f_{n_{k}}\right\|_{X} \leq C D\left\|\left(c_{k}\right)\right\|_{c_{0}} .
$$

Theorem 4.6 Let $\varphi$ be an increasing concave function on $[0, \infty)$ such that

$$
\lim _{t \rightarrow 0^{+}} \varphi(t)=0 \text { and } q_{\varphi}<1 .
$$


Then the space $X=C\left(M_{\varphi}^{0}\right)$ on $[0, \infty)$ has the Dunford-Pettis property.

Proof Assuming to the contrary that $X$ does not have the Dunford-Pettis property, we can find sequences $\left\{u_{n}\right\} \subset X$ such that $\left\|u_{n}\right\|_{X}=1, u_{n} \rightarrow 0$ weakly in $X$, and $\left\{v_{n}\right\} \subset X^{*}=X^{\prime}=\widetilde{\Lambda_{\psi}}$ such that $\left\|v_{n}\right\|_{X^{\prime}}=1, v_{n} \rightarrow 0$ weakly in $X^{\prime}$ satisfying the condition

$$
\left\langle u_{n}, v_{n}\right\rangle:=\int_{0}^{\infty} u_{n}(t) v_{n}(t) d t \geq \delta
$$

for some $\delta>0$ and all $n \in \mathbb{N}$. It is easy to see that $u_{n} \chi_{[a, b]} \rightarrow 0$ weakly in $X$ for every $0<a<b<\infty$. In fact, if $v \in X^{\prime}$, then $\left\langle u_{n} \chi_{[a, b]}, v\right\rangle=\left\langle u_{n}, v \chi_{[a, b]}\right\rangle \rightarrow 0$ as $n \rightarrow \infty$, because of $v \chi_{[a, b]} \in X^{\prime}$. Moreover (see Proposition 2.2),

$$
\left.X\right|_{[a, b]}:=\{u \in X: \operatorname{supp} u \subset[a, b]\}=L_{1}[a, b]
$$

with equivalence of norms, and therefore $u_{n} \chi_{[a, b]} \rightarrow 0$ weakly in $L_{1}[a, b]$. Setting $\alpha_{n}(u):=\int_{a}^{b} u(t) v_{n}(t) d t, n \in \mathbb{N}$, we see that $\alpha_{n} \in\left(L_{1}[a, b]\right)^{*}=L_{\infty}[a, b]=X^{\prime} / M$, where $M=\left\{v \in X^{\prime}:\langle u, v\rangle=0\right.$ for all $\left.u \in L_{1}[a, b]\right\}$. Then

$$
\left(L_{\infty}[a, b]\right)^{*}=\left(X^{\prime} / M\right)^{*}=\left\{F \in\left(X^{\prime}\right)^{*}: F(v)=0 \text { for all } v \in M\right\},
$$

and therefore $\left(L_{\infty}[a, b]\right)^{*} \subset\left(X^{\prime}\right)^{*}$. Hence, from the fact that $v_{n} \rightarrow 0$ weakly in $X^{\prime}$, it follows that $\alpha_{n} \rightarrow 0$ weakly in $L_{\infty}[a, b]$. Since $L_{1}[a, b]$ has the Dunford-Pettis property, as a result we have

$$
\alpha_{n}\left(u_{n} \cdot \chi_{[a, b]}\right)=\int_{a}^{b} u_{n}(t) v_{n}(t) d t \rightarrow 0 \quad \text { as } n \rightarrow \infty,
$$

for every $0<a<b<\infty$. Thus, taking into account (4.10), we can select subsequences of $\left\{u_{n}\right\}$ and $\left\{v_{n}\right\}$ (we will denote them still by $\left\{u_{n}\right\}$ and $\left\{v_{n}\right\}$ ) such that at least one of the following conditions holds

- there exists a sequence $\left\{b_{n}\right\}_{n=1}^{\infty}$ with $b_{1}>b_{2}>\cdots, \lim _{n \rightarrow \infty} b_{n}=0$ and

$$
\int_{0}^{b_{n}} u_{n}(t) v_{n}(t) d t \geq \frac{3 \delta}{4}, \quad n \in \mathbb{N} ;
$$

- there exists a sequence $\left\{a_{n}\right\}_{n=1}^{\infty}$ with $a_{1}<a_{2}<\cdots, \lim _{n \rightarrow \infty} a_{n}=\infty$ and

$$
\int_{a_{n}}^{\infty} u_{n}(t) v_{n}(t) d t \geq \frac{3 \delta}{4}, \quad n \in \mathbb{N} .
$$

Since $\int_{0}^{\infty}\left|u_{n}(t) v_{n}(t)\right| d t<\infty$ for every $n \in \mathbb{N}$, passing to further subsequences, we can find a sequence of intervals $I_{n}=\left[a_{n}, b_{n}\right], n=1,2, \ldots$ such that either $b_{1}>a_{1}>$ $b_{2}>a_{2}>\cdots, \lim _{n \rightarrow \infty} b_{n}=0$, or $a_{1}<b_{1}<a_{2}<b_{2}<\cdots, \lim _{n \rightarrow \infty} a_{n}=\infty$, for which

$$
\int_{I_{n}} u_{n}(t) v_{n}(t) d t \geq \frac{\delta}{2}, \quad n \in \mathbb{N} .
$$

Now we set $f_{n}:=u_{n} \cdot \chi_{I_{n}}, n=1,2, \ldots$ From (4.11) it follows that $\left\{f_{n}\right\}$ is a seminormalized sequence in $X$. So applying Corollary 4.5 , we can extract a subsequence (denoted still by $\left\{f_{n}\right\}$ ), which is equivalent in $X$ to the canonical basis in $c_{0}$. Therefore, $f_{n} \rightarrow 0$ weakly in the closed linear span $\left[f_{n}\right]$ (and in $X$ ). Clearly, $\theta_{n}(f):=$ $\int_{0}^{\infty} f(t) v_{n}(t) d t$ is a bounded linear functional on $\left[f_{n}\right]$. As above, $\left[f_{n}\right]^{* *} \subset\left(X^{\prime}\right)^{*}$. 
Therefore, since $v_{n} \rightarrow 0$ weakly in $X^{\prime}$, we have $\theta_{n} \rightarrow 0$ weakly in $\left[f_{n}\right]^{*}$. Noting that the subspace $\left[f_{n}\right]$ is isomorphic to $c_{0}$, which has the Dunford-Pettis property, we obtain

$$
\int_{I_{n}} u_{n}(t) v_{n}(t) d t=\theta_{n}\left(f_{n}\right) \rightarrow 0 \quad \text { as } n \rightarrow \infty,
$$

which contradicts (4.11). Thus, the proof is complete.

As we know, the condition $0<p_{\varphi} \leq q_{\varphi}<1$ guarantees that $C \Lambda_{\varphi}$ on $[0, \infty)$ is a weighted $L_{1}$-space up to equivalence of norms (see [DS07, Theorem 4.4], [LM15a]). It turns out that a similar result holds also for the Cesàro-Lorentz spaces on $[0,1]$.

Theorem 4.7 Let $\varphi$ be an increasing concave function on $[0,1]$ such that $0<p_{\varphi}^{0} \leq$ $q_{\varphi}^{0}<1$. Then $C \Lambda_{\varphi}[0,1]=L_{1}(w)$, with $w(t)=\int_{0}^{1-t} \frac{\varphi^{\prime}(s)}{t+s} d s$.

Proof By duality and Fubini's theorem, we have

$$
\begin{aligned}
\|f\|_{C \Lambda_{\varphi}} & =\sup _{\|g\|_{\Lambda_{\varphi}^{\prime} \leq 1}} \int_{0}^{1} C|f|(x)|g(x)| d x=\sup _{\|g\|_{\Lambda_{\varphi}^{\prime} \leq 1}} \int_{0}^{1}|g(x)|\left(\frac{1}{x} \int_{0}^{x}|f(t)| d t\right) d x \\
& =\sup _{\|g\|_{\Lambda_{\varphi}^{\prime} \leq 1}} \int_{0}^{1}|f(t)|\left(\int_{t}^{1} \frac{|g(x)|}{x} d x\right) d t \leq \int_{0}^{1}|f(t)|\left\|h_{t}\right\|_{\Lambda_{\varphi}} d t,
\end{aligned}
$$

where $h_{t}(x)=\frac{1}{x} \chi_{[t, 1]}(x)$. Then

$$
\left\|h_{t}\right\|_{\Lambda_{\varphi}}=\int_{0}^{1}\left(h_{t}\right)^{*}(s) \varphi^{\prime}(s) d s=\int_{0}^{1-t} \frac{\varphi^{\prime}(s)}{s+t} d s=w(t)
$$

and consequently the above inequality means that $L_{1}(w) \stackrel{1}{\rightarrow} C \Lambda_{\varphi}$. In view of the conditions imposed on the indices $p_{\varphi}^{0}$ and $q_{\varphi}^{0}$, the operators $C$ and $C^{*}$ are bounded in $\Lambda_{\varphi}$ (see [KPS82, Chapter II, \$8.6]). Therefore, the reverse inclusion is equivalent by duality (see Theorem 2.1 (ii)) to the following one: $L_{\infty}(1 / w) \hookrightarrow \widehat{M_{\psi}(v)}$, where $\psi(t)=$ $\frac{t}{\varphi(t)}$ and $v(t)=\frac{1}{1-t}$. Thus, it is enough to check that $w \in \overline{M_{\psi}(v)}$, i.e.,

$$
\|w\|_{\overline{M_{\psi}(v)}}=\sup _{0<t \leq 1} \frac{1}{\varphi(t)} \int_{0}^{t}(v \widetilde{w})^{*}(x) d x<\infty .
$$

First notice that $w$ is decreasing, so we have $\widetilde{w}=w$. We divide the function $v \cdot w$ into two parts, namely,

$$
v(t) w(t)=\frac{w(t)}{1-t} \chi_{[0,1 / 2]}(t)+\frac{w(t)}{1-t} \chi_{[1 / 2,1]}(t)=w_{0}(t)+w_{1}(t) .
$$

Thus, we need only to check that $w_{0}$ and $w_{1}$ belong to the space $M_{\psi}$.

By Fubini's theorem, we have

$$
\begin{aligned}
\int_{0}^{x} w^{*}(t) d t & =\int_{0}^{x}\left(\int_{0}^{1-t} \frac{\varphi^{\prime}(s)}{t+s} d t\right) d s \\
& =\int_{0}^{1-x}\left(\int_{0}^{x} \frac{\varphi^{\prime}(s)}{t+s} d t\right) d s+\int_{1-x}^{1}\left(\int_{0}^{1-s} \frac{\varphi^{\prime}(s)}{t+s} d t\right) d s \\
& =\int_{0}^{1-x} \varphi^{\prime}(s) \ln \frac{x+s}{s} d s+\int_{1-x}^{1} \varphi^{\prime}(s) \ln \frac{1}{s} d s .
\end{aligned}
$$


Then, since $0<x<1 / 2$, the second summand can be estimated thanks to monotonicity and subadditivity of the concave function $\varphi$ as follows:

$$
\int_{1-x}^{1} \varphi^{\prime}(s) \ln \frac{1}{s} d s \leq \ln 2 \int_{1-x}^{1} \varphi^{\prime}(s) d s=\ln 2[\varphi(1)-\varphi(1-x)] \leq \ln 2 \varphi(x) .
$$

While for the first one, integrating by parts we get

$$
\begin{aligned}
\int_{0}^{1-x} \varphi^{\prime}(s) \ln \frac{x+s}{s} d s= & \varphi(1-x) \ln \frac{1}{1-x}-\lim _{s \rightarrow 0^{+}} \varphi(s) \ln \frac{x+s}{s} \\
& +x \int_{0}^{1-x} \frac{\varphi(s)}{(s+x) s} d s \\
\leq & \varphi(1) \frac{x}{1-x}+x \int_{0}^{1-x} \frac{\varphi(s)}{(s+x) s} d s .
\end{aligned}
$$

Since $0<x<1 / 2$, then by concavity of $\varphi$, we get $\varphi(1) \frac{x}{1-x} \leq 2 \varphi(1) x \leq 2 \varphi(x)$. Moreover, for some $0<a<1, A \geq 1$, and all $0<x<1, t>0$ we have $\varphi(t x) \leq A t^{a} \varphi(x)$ and consequently, putting $s=t x$, we obtain

$$
\begin{aligned}
\int_{0}^{1-x} \frac{\varphi(s)}{(s+x) s} d s & =\frac{1}{x} \int_{0}^{\frac{1-x}{x}} \frac{\varphi(t x)}{(1+t) t} d t \leq \frac{A}{x} \int_{0}^{\frac{1-x}{x}} \frac{t^{a} \varphi(x)}{(1+t) t} d t \\
& \leq A \frac{\varphi(x)}{x} \int_{0}^{\infty} \frac{t^{a-1}}{1+t} d t=B \frac{\varphi(x)}{x} .
\end{aligned}
$$

Thus, for $0<x<1 / 2$,

$$
\begin{aligned}
\int_{0}^{x}\left(w_{0}\right)^{*}(t) d t & \leq 2 \int_{0}^{x}\left(w \chi_{(0,1 / 2]}\right)^{*}(t) d t \leq 2 \int_{0}^{x} w^{*}(t) d t \\
& \leq 2(2+B+\ln 2) \varphi(x)
\end{aligned}
$$

whence $w_{0} \in M_{\psi}$.

Let us consider now $w_{1}$. For $1 / 2<t \leq 1$ we have

$$
w_{1}(t)=\frac{1}{1-t} \int_{0}^{1-t} \frac{\varphi^{\prime}(s)}{t+s} d s \leq \frac{2}{1-t} \int_{0}^{1-t} \varphi^{\prime}(s) d s=2 \frac{\varphi(1-t)}{1-t} .
$$

Since the function $\frac{\varphi(1-t)}{1-t}$ is increasing, we conclude that $w_{1}^{*}(t) \leq 2 \frac{\varphi(t)}{t}, 0<t \leq 1$. In consequence, from (2.5) and the condition $q_{\psi}^{0}=1-p_{\varphi}^{0}<1$, it follows that

$$
\int_{0}^{x} w_{1}^{*}(t) d t \leq 2 \int_{0}^{x} \frac{\varphi(t)}{t} d t=2 \int_{0}^{x} \frac{1}{\psi(t)} d t \leq 2 C \frac{x}{\psi(x)}=2 C \varphi(x),
$$

which finishes the proof.

Of course, from Theorem 4.7 it follows that the space $C \Lambda_{\varphi}[0,1]$ has the DunfordPettis property whenever $0<p_{\varphi}^{0} \leq q_{\varphi}^{0}<1$. Let us prove an analogous result for separable Cesàro-Marcinkiewicz spaces.

Theorem 4.8 Let $\varphi$ be an increasing concave function on $[0,1]$ such that

$$
\lim _{t \rightarrow 0^{+}} \varphi(t)=0 \text { and } q_{\varphi}^{0}<1 .
$$

Then the space $C\left(M_{\varphi}^{0}\right)[0,1]$ has the Dunford-Pettis property. 
Proof For every $k=2,3, \ldots$, we set

$$
X_{k}:=\left.C M_{\varphi}^{0}\right|_{[0,1-1 / k]}=\left\{f \in C M_{\varphi}^{0}: \operatorname{supp} f \subset\left[0,1-\frac{1}{k}\right]\right\} .
$$

Since $C M_{\varphi}^{0}$ is an order continuous space, the union $\bigcup_{k=2}^{\infty} X_{k}$ is dense in it. Moreover, from the definition of Cesàro spaces, it follows that, for every $k=2,3, \ldots, X_{k}$ can be regarded as a complemented subspace of the space $C M_{\varphi_{1}}^{0}[0, \infty)$, where $\varphi_{1}$ is a concave extension of the function $\varphi$ to the semi-axis $[0, \infty)$ such that $q_{\varphi_{1}}<1$. (Notice that $C X[0,1]$ is not a restriction of $C X[0, \infty)$ to the interval $[0,1]$. More precisely, similarly to $\left[\mathrm{AM} 09\right.$, Remark 5], one can check that $\left.\left.C X[0, \infty)\right|_{[0,1]}=C X[0,1] \cap L_{1}[0,1]\right)$. Therefore, an inspection of the proof of Theorem 4.1 shows that

$$
X_{k} \simeq\left(\bigoplus_{n \in \mathbb{N}} L_{1}[0,1]\right) \ell_{\infty},
$$

whence the space $\left(\oplus_{k=2}^{\infty} X_{k}\right)_{\ell_{\infty}}$ is isomorphic to the latter $\ell_{\infty}-$ sum as well. Thus, $\left(\oplus_{k=2}^{\infty} X_{k}\right)_{\ell_{\infty}}$ has the Dunford-Pettis property. Finally, applying [Bo81, Proposition 2], we conclude that $C M_{\varphi}^{0}$ also possesses the latter property, and the proof is complete.

Remark 2 The assertion of Theorem 4.8 cannot be deduced from Theorem 4.1 (i), using Bourgain's results as above, because of the difference in the duality results for Cesàro spaces for the cases of $[0,1]$ and $[0, \infty)$ (see Theorem 2.1 ). We would like to mention here also that we could not identify conditions under which the space $C M_{\varphi}[0,1]$ has the Dunford-Pettis property.

Now we present some negative results related to the Dunford-Pettis property of Cesàro and Tandori function spaces.

Theorem 4.9 Let X be a reflexive symmetric function space on I such that the operator $C$ is bounded on $X$.

(i) If $I=[0, \infty)$, then the spaces $C X$ and $\widetilde{X}^{\prime}$ do not have the Dunford-Pettis property.

(ii) If $I=[0,1]$, X has the Fatou property, and the operator $C^{*}$ is bounded on $X$, then the spaces $C X$ and $\widetilde{X}^{\prime}$ do not have the Dunford-Pettis property.

Proof (i) The proof is rather similar to the proof in the sequence case (Theorem 3.7). Again it is sufficient to prove that the operator $C: C X \rightarrow X$ is not a Dunford-Pettis operator. Let us show that $x_{n}=\frac{1}{\varphi_{X}(1 / n)} \chi_{[0,1 / n]}, n=1,2, \ldots$, is a weakly null sequence in $C X$. Since $X$ is order continuous, it follows that $C X$ is also order continuous and by Theorem 2.1 (i) we obtain $(C X)^{*}=(C X)^{\prime}=\widetilde{X}^{\prime}$. Thus, we need only to check that

$$
\left\langle y, x_{n}\right\rangle=\frac{1}{\varphi_{X}(1 / n)} \int_{0}^{1 / n} y(t) d t \rightarrow 0 \quad \text { as } n \rightarrow \infty,
$$

for every decreasing positive function $y \in X^{\prime}$. Again $X^{\prime} \subset M_{\varphi_{X^{\prime}}}$, where $M_{\varphi_{X^{\prime}}}$ is the Marcinkiewicz function space with the fundamental function $\varphi_{X^{\prime}}$. By reflexivity of $X$ we have $X^{\prime}=\left(X^{\prime}\right)^{0}$, and thus

$$
X^{\prime} \subset M_{\varphi_{X^{\prime}}}^{0} \subset\left\{z=z(t): \lim _{t \rightarrow 0} \frac{\varphi_{X^{\prime}}(t)}{t} \int_{0}^{t} z^{*}(s) d s=0\right\} .
$$


But $\varphi_{X}(t)=t / \varphi_{X^{\prime}}(t)$ and (4.12) follows from the above embedding. On the other hand, $C x_{n} \geq x_{n}$ and so $\left\|C x_{n}\right\|_{X} \geq\left\|x_{n}\right\|_{X}=1$. This means that $C X$ does not have the Dunford-Pettis property. Moreover, since $\widetilde{X}^{\prime}=(C X)^{\prime}=(C X)^{*}, \widetilde{X}^{\prime}$ fails to have the latter property as well.

(ii) The only difference of this case from the case of $[0, \infty)$ is the fact that now $\left.(C X)^{*}=(C X)^{\prime}=X^{\prime}(\overline{1 /(1-t})\right)$. However, "near zero", the latter space coincides with the space $\widetilde{X}^{\prime}$ without a weight. Thus, we can repeat the same proof as in (i).

As we know [DS07, Theorem 4.4] and Theorem 4.7, the Cesàro-Lorentz spaces may coincide with weighted $L_{1}$-spaces and therefore may be isomorphic to the symmetric space $L_{1}$. At the same time, this is not the case for Cesàro spaces $C X$ when $X$ is reflexive.

Corollary 4.10 If $X$ is a reflexive symmetric function space on $[0,1]$ such that the operator $C$ is bounded on $X$, then $C X$ is not isomorphic to any symmetric space on $[0,1]$.

Proof Suppose $C X$ is isomorphic to some symmetric space $Y$ on $[0,1]$. Hence, by Proposition 2.2, $Y$ contains a complemented copy of $L_{1}[0,1]$. On the other hand, as Kalton proved [Ka93, Theorem 7.4], every separable symmetric space on $[0,1]$ that contains a complemented subspace isomorphic to $L_{1}[0,1]$ is isomorphic to $L_{1}[0,1]$ itself. Therefore, we conclude that $Y$ is isomorphic to $L_{1}[0,1]$. On the other hand, $C X \simeq Y$ cannot be isomorphic to $L_{1}[0,1]$, because by Theorem 4.9 that space fails to have the Dunford-Pettis property.

\section{Isomorphism Between $\mathrm{Ces}_{\infty}$ and $\operatorname{ces}_{\infty}$}

In [AM09, Theorem 9] (see also [AM14, Theorem 7.2]) it was proved that the spaces $\operatorname{Ces}_{\infty}[0,1]$ and $\operatorname{Ces}_{\infty}[0, \infty)$ are isomorphic and there the question was raised if the spaces $\mathrm{Ces}_{\infty}$ and $\operatorname{ces}_{\infty}$ are isomorphic [AM09, Problem 1], [AM14, Problem 4]. The following theorem answers this question in the affirmative.

Theorem 5.1 The spaces $\mathrm{Ces}_{\infty}$ and $\mathrm{ces}_{\infty}$ are isomorphic.

Proof At first, we recall that, by Corollary 3.5, ces $_{\infty} \simeq\left(\oplus_{n=0}^{\infty} \ell_{1}^{2^{n}}\right)_{\ell_{\infty}}$ and, by Theorem 4.1, $\operatorname{Ces}_{\infty} \simeq\left(\oplus_{n \in \mathbb{N}} L_{1}[0,1]\right)_{\ell_{\infty}}$. Therefore, $\operatorname{ces}_{\infty} \simeq \operatorname{ces}_{\infty} \oplus \operatorname{ces}_{\infty}$ and $\operatorname{Ces}_{\infty} \simeq$ $\operatorname{Ces}_{\infty} \oplus \operatorname{Ces}_{\infty}$, which shows that we can apply Pełczyński decomposition argument, see [Pe60, Proposition 4] or [AK06, Theorem 2.2.3]. In other words, the proof will be completed once we check that $\mathrm{ces}_{\infty}$ is isomorphic to a complemented subspace of $\mathrm{Ces}_{\infty}$ and vice versa.

Clearly, for every $n=0,1,2, \ldots$ the space $\ell_{1}^{2^{n}}$ can be complementedly embedded into the space $L_{1}[0,1]$. Therefore, the fact that ces $s_{\infty}$ is isomorphic to a complemented subspace of $\mathrm{Ces}_{\infty}$ follows at once from the above isomorphic representations of these spaces.

Just a little more effort is required for the proof of the reverse statement. Let us represent the set $\mathbb{N} \cup\{0\}$ as a union of infinite increasing pairwise disjoint sequences 
$\left(a_{n}^{k}\right)_{n=0}^{\infty}, k=1,2, \ldots$ Then we can write

$$
\operatorname{ces}_{\infty} \simeq\left(\bigoplus_{k=1}^{\infty}\left(\bigoplus_{n=0}^{\infty} \ell_{1}^{2^{a_{n}^{k}}}\right)_{\infty}\right)_{l_{\infty}}
$$

Since $n \leq a_{n}^{k}$, where $k=1,2, \ldots$ and $n=0,1,2, \ldots$ are arbitrary, the space $\ell_{1}^{2^{n}}$ can be considered as a complemented subspace of the space $\ell_{1}^{2^{a_{n}^{k}}}$. Let $P_{n}^{k}$ be a respective projection and $P_{k}=\oplus_{n=0}^{\infty} P_{n}^{k}$. Noting that $P_{k}\left(\left(\oplus_{n=0}^{\infty} \ell_{1}^{2^{a_{n}^{k}}}\right)_{\ell_{\infty}}\right)=\left(\oplus_{n=0}^{\infty} \ell_{1}^{2^{n}}\right)_{\ell_{\infty}}$, we see that by Theorem 3.7, $L_{1}[0,1]$ is complemented in $P_{k}\left(\left(\oplus_{n=0}^{\infty} \ell_{1}^{2^{a_{n}^{k}}}\right)_{\ell_{\infty}}\right)$ and hence in the space $\left(\oplus_{n=0}^{\infty} \ell_{1}^{2^{a_{n}^{k}}}\right) \ell_{\infty}$. Finally, from (5.1) it follows that $\operatorname{Ces}_{\infty} \simeq\left(\oplus_{n \in \mathbb{N}} L_{1}[0,1]\right)_{\infty}$ is isomorphic to a complemented subspace of $\operatorname{ces}_{\infty}$ and the proof is complete.

Corollary 5.2 The space $\operatorname{Ces}_{\infty}(I)$, where $I=[0, \infty)$ or $[0,1]$, is isomorphic to a dual space.

Proof By (2.9) and Theorem 5.1 we have $\left(\widetilde{\ell_{1}}\right)^{*}=\left(\widetilde{\ell_{1}}\right)^{\prime}=\operatorname{ces}_{\infty} \simeq \operatorname{Ces}_{\infty}$.

In contrast to the latter result, order continuous Cesàro spaces fail to be isomorphic to the dual ones.

Proposition 5.3 If $X$ is a symmetric function space on $I=[0,1]$ or $I=[0, \infty)$ such that $X$ is order continuous and $C$ is bounded on $X$, then $C X$ is not isomorphic to a dual space.

Proof Suppose that $C X$ is isomorphic to a dual space. By Proposition 2.2, $C X$ contains a complemented subspace isomorphic to $L_{1}[0,1]$. Therefore, applying the Hagler-Stegall theorem [HS73, Theorem 1], we see that CX also contains a subspace isomorphic to $C[0,1]^{*}$. However, it is impossible, since, by [LM15b, Lemma 1], $C X$ is separable.

Let us comment on the latter results. Suppose that $X$ is an ideal Banach function space with the Fatou property such that the separable part of its Köthe dual $\left(X^{\prime}\right)^{0}$ has the same support as $X$ itself. Then an easy argument shows that

$$
\left[\left(X^{\prime}\right)^{0}\right]^{*}=\left[\left(X^{\prime}\right)^{0}\right]^{\prime}=X^{\prime \prime}=X,
$$

i.e., $X$ is a dual space. So the space $\left(X^{\prime}\right)^{0}$ is a natural candidate for being the predual of a dual ideal Banach space $X$. Moreover, as we have seen, separable $C X$ spaces are not isomorphic to dual ones similarly to $L_{1}$ and both of them have Köthe dual without nontrivial absolutely continuous elements. Hence, the following conjecture may arise: an ideal Banach space whose Köthe dual has trivial subspace of order continuous elements is not isomorphic to a dual space. This statement, however, is false; by Corollary 5.2, the Cesàro space $\operatorname{Ces}_{\infty}$, satisfying $\left[\left(\operatorname{Ces}_{\infty}\right)^{\prime}\right]^{0}=\left(\widetilde{L_{1}}\right)^{0}=\{0\}$, is a dual space. In contrast to that, the symmetric space $X=L_{1}+L_{\infty}$ on $[0, \infty)$, for which we also have $\left(X^{\prime}\right)^{0}=\left(L_{1} \cap L_{\infty}\right)^{0}=\{0\}$, is not isomorphic to a dual space [AM17, Theorem 4].

It is interesting to observe that the above phenomenon has its counterpart in the general theory of Banach lattices. Let $E$ be a separable Banach lattice satisfying the 
Radon-Nikodym property (RNP). Then the set $F$ of all $x^{*} \in E^{*}$, such that the interval $\left[0,\left|x^{*}\right|\right]$ is weakly compact, is a Banach lattice. Lotz showed (in an unpublished preprint [Lo75]) that if $F$ is big enough, i.e., the topology $\sigma(E, F)$ is Hausdorff, then $E=F^{*}$. Hence, $F$ is a natural candidate as the predual of $E$. Talagrand, however, motivated by above results, constructed a separable Banach lattice being a dual space (and hence with RNP) such that for each $x^{*} \in E^{*}, x^{*} \geq 0$, the interval $\left[0, x^{*}\right]$ is not weakly compact [Ta81].

To see that the space $\mathrm{Ces}_{\infty}$ may be regarded as a natural "function" counterpart of Talagrand's example (which seems to be rather artificial) we present the following simple assertion.

Proposition 5.4 Let $X$ be an ideal Banach space on $[0,1], x_{0} \in X, x_{0} \geq 0$. Then the interval $\left[0, x_{0}\right]$ is weakly compact in $X$ if and only if $x_{0} \in X^{0}$.

Proof First let $\left[0, x_{0}\right]$ be weakly compact in $X$. On the contrary, assume that $x_{0} \notin$ $X^{0}$. Then there is a sequence of sets $\left\{A_{n}\right\}_{n=1}^{\infty}, A_{1} \supset A_{2} \supset A_{3} \supset \cdots, \cap_{n=1}^{\infty} A_{n}=\varnothing$, and $\varepsilon>0$ such that

$$
\left\|x_{0} \chi_{A_{n}}\right\|_{X} \geq \varepsilon
$$

Since, by hypothesis, $x_{0} \chi_{A_{n}} \in\left[0, x_{0}\right]$, we can find a subsequence $\left\{x_{0} \chi_{A_{n_{k}}}\right\}_{k=1}^{\infty}$ such that $x_{0} \chi_{A_{n_{k}}} \rightarrow y$ weakly in $X$. Then, by [KA77, Lemma 10.4.1], we get that

$$
\left\|x_{0} \chi_{A_{n_{k}}}\right\|_{X} \rightarrow 0 \text { as } k \rightarrow \infty \text {. }
$$

This contradicts (5.2).

Conversely, let $x_{0} \in X^{0}$. Clearly, we have $\left[0, x_{0}\right] \subset X^{0}$. Therefore, by [KA77, Lemma 10.4.2], the interval $\left[0, x_{0}\right]$ is weakly compact in $X^{0}$, i.e., with respect to the topology generated in $X^{0}$ by the space $\left(X^{0}\right)^{*}=X^{\prime}$. Since $X^{*}=X^{\prime} \oplus X_{s}^{\prime}$, where $X_{s}^{\prime}$ consists of all singular functionals $f$ such that $\left.f\right|_{X^{0}}=0$ [KA77, Theorem 10.3.6], we get that $\left[0, x_{0}\right]$ is weakly compact in $X$ as well.

Remark 3 In particular, from Proposition 5.4, it follows that Lotz's result cannot be applied to $\operatorname{Ces}_{\infty}$. In fact, $\left(\operatorname{Ces}_{\infty}\right)^{*}=\widetilde{L_{1}} \oplus S$, where $S$ is the space of singular functionals, and, since singular functionals are not comparable with regular ones, each interval $\left[0,\left|x^{*}\right|\right] \subset \operatorname{Ces}_{\infty}^{*}$ is either non-weakly compact or is of the form $[0,|s|]$ with $s \in S$. Therefore, the set $F$ of all $x^{*} \in\left(\operatorname{Ces}_{\infty}\right)^{*}$ with the weakly compact interval $\left[0,\left|x^{*}\right|\right]$ is contained in $S$ and the topology $\sigma\left(\operatorname{Ces}_{\infty}, F\right)$ fails to be Hausdorff, because singular functionals vanish on absolutely continuous elements.

One more interesting observation comes from the above considerations.

Remark 4 The space $\left(\oplus_{k=1}^{\infty} L_{1}[0,1]\right)_{\ell_{\infty}}$ is isomorphic to a dual space, but its unit sphere does not contain extreme points.

Since $\operatorname{Ces}_{\infty}(I) \simeq X^{*}$, where $X$ is a Banach space, and it contains a complemented subspace isomorphic to $L_{1}[0,1]$, then, according to the above-mentioned HaglerStegall result, $\operatorname{Ces}_{\infty}(I)$ contains a complemented subspace isomorphic to $C[0,1]^{*}$, i.e., to the space $\mathcal{M}[0,1]$ of all regular Borel measures on $[0,1]$ of finite variation. We 
would like to conclude this paper by presenting the following stronger result, which was noticed by Michał Wojciechowski and is included here with his kind permission.

Theorem 5.5 The spaces $\operatorname{Ces}_{\infty}(I)$, where $I=[0,1]$ or $[0, \infty)$, is isomorphic to the space $\left(\oplus_{k=1}^{\infty} \mathcal{M}[0,1]\right)_{\ell_{\infty}}$.

Proof At first, by Miljutin's theorem [AK06, p. 94], we know that $C[0,1] \simeq C(\mathbb{T})$, where $\mathbb{T}$ is the unit circle. Since also $\mathcal{M}[0,1] \simeq \mathcal{M}(\mathbb{T})$ and $L_{1}[0,1] \simeq L_{1}(\mathbb{T})$, we can regard all spaces on $\mathbb{T}$ instead of $[0,1]$.

By Theorem 4.1, it is sufficient to prove that the spaces

$$
\left(\bigoplus_{k=1}^{\infty} L_{1}(\mathbb{T})\right)_{\ell_{\infty}} \text { and }\left(\bigoplus_{k=1}^{\infty} \mathcal{M}(\mathbb{T})\right)_{\ell_{\infty}}
$$

are isomorphic. Since both spaces are isomorphic to their squares, we may again apply the Pełczyński decomposition argument. Clearly, $\left(\oplus_{k=1}^{\infty} L_{1}(\mathbb{T})\right)_{\ell_{\infty}}$ is isomorphic to a complemented subspace of $\left(\oplus_{k=1}^{\infty} \mathcal{M}(\mathbb{T})\right)_{\ell_{\infty}}$. So we only need to check that, conversely, $\left(\oplus_{k=1}^{\infty} \mathcal{M}(\mathbb{T})\right)_{\ell_{\infty}}$ is isomorphic to a complemented subspace of

$$
\left(\bigoplus_{k=1}^{\infty} L_{1}(\mathbb{T})\right)_{\ell_{\infty}}
$$

Let $\left\{K_{n}\right\}_{n=1}^{\infty}$ be the Fejer kernel and let $\left\{N_{i}\right\}_{i=1}^{\infty}$ be a sequence of pairwise disjoint infinite subsets of positive integers such that $\sum_{i=1}^{\infty} N_{i}=\mathbb{N}$. For every $i=1,2, \ldots$, define the operator $K^{i}: \mathcal{M}(\mathbb{T}) \rightarrow\left(\oplus_{k=1}^{\infty} L_{1}(\mathbb{T})\right)_{\ell_{\infty}}$ as follows:

$$
K^{i}(\mu):=\left(K_{n} * \mu\right)_{n \in N_{i}} \text { for every } \mu \in \mathcal{M}(\mathbb{T}) \text {. }
$$

Then $\left\|K^{i}\right\|=1$, and if $N_{i}=\left\{n_{j}^{i}\right\}_{j=1}^{\infty}, n_{1}^{i}<n_{2}^{i}<\cdots$, then $K_{n_{j}^{i}} * \mu \rightarrow \mu$ as $j \rightarrow \infty$ weakly ${ }^{*}$ in $\mathcal{M}(\mathbb{T})$ for each $i=1,2, \ldots$. Hence, $K:=\bigoplus_{i=1}^{\infty} K^{i}$ is an injective operator from $\left(\oplus_{i=1}^{\infty} \mathcal{M}(\mathbb{T})\right)_{\ell_{\infty}}$ into the space

$$
\left(\bigoplus_{k=1}^{\infty} L_{1}(\mathbb{T})\right)_{\ell_{\infty}} \simeq\left(\bigoplus_{i=1}^{\infty}\left(\bigoplus_{n \in N_{i}} L_{1}(\mathbb{T})\right)_{\ell_{\infty}}\right)_{\ell_{\infty}}
$$

Denoting by $Y$ the image of $K$, we prove that it is complemented in the latter space.

Let $\mathcal{U}$ be a free ultrafilter. For a given $\left\{f_{k}\right\} \in\left(\oplus_{k=1}^{\infty} L_{1}(\mathbb{T})\right)_{\ell_{\infty}}$ and any $i=1,2, \ldots$ define the functional $g_{i}^{*} \in C(\mathbb{T})^{*}$ by $\left\langle g_{i}^{*}, g\right\rangle:=\lim _{\mathcal{u}}\left\langle f_{n_{j}^{i}}, g\right\rangle, g \in C(\mathbb{T})$. Since $\left\|\left\{f_{k}\right\}\right\|=$ $\sup _{k \in \mathbb{N}}\left\|f_{k}\right\|_{L_{1}}<\infty$, then $g_{i}^{*}$ is a well-defined linear and bounded functional. Therefore, by the Riesz representation theorem, for every $i=1,2, \ldots$, there is a measure $\mu_{i} \in \mathcal{M}(\mathbb{T})$ such that $\left\langle g_{i}^{*}, g\right\rangle=\left\langle\mu_{i}, g\right\rangle$ for each $g \in C(\mathbb{T})$. Setting $P\left(\left\{f_{k}\right\}\right):=\left\{\mu_{i}\right\}$, we see that $P$ is a linear bounded operator from $\left(\oplus_{k=1}^{\infty} L_{1}(\mathbb{T})\right)_{\ell_{\infty}}$ into $\left(\oplus_{k=1}^{\infty} \mathcal{M}(\mathbb{T})\right)_{\ell_{\infty}}$. It remains only to show that the composition $K P$ is a projection from $\left(\oplus_{k=1}^{\infty} L_{1}(\mathbb{T})\right)_{\ell_{\infty}}$ onto $Y$. In fact, suppose that $\left\{f_{k}\right\} \subset Y$. Then $f_{n_{j}^{i}}=K_{n_{j}^{i}} * \mu_{i}, i, j=1,2, \ldots$, where $\left\{\mu_{i}\right\} \in\left(\oplus_{i=1}^{\infty} \mathcal{M}(\mathbb{T})\right)_{l_{\infty}}$, and we have

$$
\lim _{\mathcal{U}}\left\langle f_{n_{j}^{i}}, g\right\rangle=\lim _{\mathcal{U}}\left\langle K_{n_{j}^{i}} * \mu_{i}, g\right\rangle=\lim _{\mathcal{U}}\left\langle\mu_{i}, K_{n_{j}^{i}} * g\right\rangle=\lim _{j \rightarrow \infty}\left\langle\mu_{i}, K_{n_{j}^{i}} * g\right\rangle=\left\langle\mu_{i}, g\right\rangle,
$$

for every $g \in C(\mathbb{T})$. Thus, $K P\left\{f_{k}\right\}=\left\{f_{k}\right\}$ if $\left\{f_{k}\right\} \in Y$, and the proof is complete.

Acknowledgements The second named author is very grateful to Professors Yves Raynaud and Michał Wojciechowski for valuable suggestions, advice, and remarks 
concerning results from the last section. The authors would like to thank the referee for his/her careful reading of our manuscript, especially for a nice observation related to the proof of Theorem 3.3, which simplifies our original proof.

\section{References}

[AK06] F. Albiac and N. J. Kalton, Topics in Banach space theory. Springer-Verlag, New York 2006.

[Al57] A. Alexiewicz, On Cauchy's condensation theorem. Studia Math. 16(1957), 80-85.

http://dx.doi.org/10.4064/sm-16-1-80-85

[AB85] C. D. Aliprantis and O. Burkinshaw, Positive operators. Academic Press, New York, London 1985.

[AM08] S. V. Astashkin and L. Maligranda, Cesàro function spaces fail the fixed point property. Proc. Amer. Math. Soc. 136(2008), no. 12, 4289-4294. http://dx.doi.org/10.1090/S0002-9939-08-09599-3

[AM09] — Structure of Cesáro function spaces. Indag. Math. (N.S.) 20(2009), no. 3, 329-379. http://dx.doi.org/10.1016/S0019-3577(10)00002-9

[AM13] $\longrightarrow$ Interpolation of Cesáro sequence and function spaces. Studia Math. 215(2013), no. 1, 39-69. http://dx.doi.org/10.4064/sm215-1-4

[AM14] $\longrightarrow$ Structure of Cesàro function spaces: a survey. Banach Center Publ. 102(2014), $13-40$.

[AM17] $\longrightarrow, L_{p}+L_{\infty}$ and $L_{p} \cap L_{\infty}$ are not isomorphic for all $1 \leq p<\infty, p \neq 2$. Proc. Amer. Math. Soc. (2018), no. 5, 2181-2194. http://dx.doi.org/10.1090/proc/13928

[Ba32] S. Banach, Théorie des opérations linéaires. Monografe Matematyczne 1, Warszawa, 1932.

[Be96] G. Bennett, Factorizing classical inequalities. Mem. Amer. Math. Soc. 120(1996), no. 576.

[BS88] C. Bennett and R. Sharpley, Interpolation of operators. Academic Press, Boston, MA, 1988.

[B81] J. Bourgain, New classes of $\mathcal{L}^{p}$-spaces. Lecture Notes in Math., 889. Springer-Verlag, Berlin, 1981.

[Bo81] — On the Dunford-Pettis property. Proc. Amer. Math. Soc. 81(1981), no. 2, 265-272. http://dx.doi.org/10.1090/S0002-9939-1981-0593470-8

[CG94] J. M. Castillo and M. Gonzáles, On the Dunford-Pettis property in Banach spaces. Acta Univ. Carolin. Math. Phys. 35(1994), no. 2, 5-12.

[CM08] P. Cembranos and J. Mendoza, The Banach spaces $\ell^{\infty}\left(\ell^{1}\right)$ and $\ell^{1}\left(\ell^{\infty}\right)$ are not isomorphic. J. Math. Anal. Appl. 341(2008), no. 1, 295-297. http://dx.doi.org/10.1016/j.jmaa.2007.10.027

[CI90] C.-H. Chu and B. Iochum, The Dunford-Pettis property in $C^{*}$-algebras. Studia Math. 97(1990), 59-64. http://dx.doi.org/10.4064/sm-97-1-59-64

[CH01] Y. Cui and H. Hudzik, Packing constant for Cesàro sequence spaces. Nonlinear Anal. 47(2001), 2695-2702. http://dx.doi.org/10.1016/S0362-546X(01)00389-3

[CMP00] Y. Cui, C. Meng, and R. Płuciennik, Banach-Saks property and property ( $\beta$ ) in Cesàro sequence spaces. Southeast Asian Bull. Math. 24(2000), 201-210.

[CR16] G. P. Curbera and W. J. Ricker, Abstract Cesáro spaces: integral representations. J. Math. Anal. Appl. 441(2016), no. 1, 25-44. http://dx.doi.org/10.1016/j.jmaa.2016.03.074

[CR17] — The weak Banach-Saks property for function spaces. Rev. R. Acad. Cienc. Exactas Fís. Nat. Ser. A Math. RACSAM 111(2017), no. 3, 657-671. http://dx.doi.org/10.1007/s13398-016-0317-z

[DS07] O. Delgado and J. Soria, Optimal domain for the Hardy operator. J. Funct. Anal. 244(2007), no. 1, 119-133. http://dx.doi.org/10.1016/j.jfa.2006.12.011

[Di80] J. Diestel, A survey of results related to the Dunford-Pettis property. Contemp. Math. 2(1980), 15-60.

[GP03] A. Gogatishvili and L. Pick, Discretization and anti-discretization of rearrangement-invariant norms. Publ. Mat. 47(2003), no. 2, 311-358. http://dx.doi.org/10.5565/PUBLMAT_47203_02

[GHS96] M. L. Goldman, H. P. Heinig, and V. D. Stepanov, On the principle of duality in Lorentz spaces. Canad. J. Math. 48(1996), no. 5, 959-979. http://dx.doi.org/10.4153/CJM-1996-050-3

[GE98] K.-G. Grosse-Erdmann, The blocking technique, weighted mean operators and Hardy's inequality. Lecture Notes in Math., 1679. Springer-Verlag, Berlin, 1998.

[HS73] J. Hagler and C. Stegall, Banach spaces whose duals contain complemented subspaces isomorphic to $C[0,1]^{*}$. J. Funct. Anal. 13(1973), 233-251. http://dx.doi.org/10.1016/0022-1236(73)90033-5

[Ja74] A. A. Jagers, A note on Cesàro sequence spaces. Nieuw Arch. Wisk. 22(1974), 113-124. 
[Ka93] N. Kalton, Lattice structures on Banach spaces. Mem. Amer. Math. Soc. 103(1993), no. 493.

[KK12] A. Kamińska and D. Kubiak, On the dual of Cesàro function space. Nonlinear Analysis 75(2012), no. 5, 2760-2773. http://dx.doi.org/10.1016/j.na.2011.11.019

[KM00] A. Kamińska and M. Mastyło, The Dunford-Pettis property for symmetric spaces. Canad. J. Math. 52(2000), no. 4, 789-803. http://dx.doi.org/10.4153/CJM-2000-033-9

[KA77] L. V. Kantorovich and G. P. Akilov, Functional analysis. Nauka, Moscow 1977 (Russian); English transl. Pergamon Press, Oxford-Elmsford, New York 1982.

[KMS07] R. Kerman, M. Milman, and G. Sinnamon, On the Brudnyì-Krugljak duality theory of spaces formed by the K-method of interpolation. Rev. Mat. Complut. 20(2007), no. 2, 367-389.

[KKL48] B. I. Korenblyum, S. G. Krein, and B. Y. Levin, On certain nonlinear questions of the theory of singular integrals. Doklady Akad. Nauk SSSR (N.S.) 62(1948), 17-20 (Russian).

[KR61] M. A. Krasnoselskii and Y. B. Rutickii, Convex functions and Orlicz spaces. Noordhoff, Groningen, 1961.

[KPS82] S. G. Krein, Y. I. Petunin, and E. M. Semenov, Interpolation of linear operators. Amer. Math. Soc., Providence, RI, 1982.

[LM15a] K. Leśnik and L. Maligranda, On abstract Cesàro spaces. Duality. J. Math. Anal. Appl. 424(2015), no. 2, 932-951. http://dx.doi.org/10.1016/j.jmaa.2014.11.023

[LM15b] Abstract Cesàro spaces. Optimal range. Integral Equations Operator Theory 81(2015), no. 2, 227-235. http://dx.doi.org/10.1007/s00020-014-2203-4

[LM16] Interpolation of abstract Cesàro, Copson and Tandori spaces. Indag. Math. (N.S.) 27(2016), no. 3, 764-785. http://dx.doi.org/10.1016/j.indag.2016.01.009

[Le93] D. H. Leung, Isomorphism of certain weak $L^{p}$ spaces, Studia Math. 104(1993), no. 2, 151-160. http://dx.doi.org/10.4064/sm-104-2-151-160

[Li04] P.-K. Lin, Köthe-Bochner function spaces. Birkhäuser, Boston, 2004.

[LT77] J. Lindenstrauss and L. Tzafriri, Classical Banach spaces, I. Sequence spaces. Springer-Verlag, Berlin, 1977.

[LT79] Classical Banach spaces, II. Function spaces. Springer-Verlag, Berlin, 1979.

[Lo75] H. P. Lotz, The Radon-Nikodym property in Banach lattices. Univ. of Illinois, Urbana-Champaign, preprint, 1975.

[LZ66] W. A. J. Luxemburg and A. C. Zaanen, Some examples of normed Köthe spaces. Math. Ann. 162(1966), 337-350. http://dx.doi.org/10.1007/BF01369107

[Ma85] L. Maligranda, Indices and interpolation. Dissertationes Math. (Rozprawy Mat.) 234(1985), $1-49$.

[Ma89] Orlicz spaces and interpolation. Seminars in Mathematics 5, University of Campinas, Campinas, 1989.

[MPS07] L. Maligranda, N. Petrot, and S. Suantai, On the James constant and B-convexity of Cesàro and Cesàro-Orlicz sequence spaces. J. Math. Anal. Appl. 326(2007), no. 1, 312-331. http://dx.doi.org/10.1016/j.jmaa.2006.02.085

[MS06] M. Mastyło and G. Sinnamon, A Calderón couple of down spaces. J. Funct. Anal. 240(2006), no. 1, 192-225. http://dx.doi.org/10.1016/j.jfa.2006.05.007

[NP10] A. Nekvinda and L. Pick, Optimal estimates for the Hardy averaging operator. Math. Nachr. 283(2010), no. 2, 262-271. http://dx.doi.org/10.1002/mana.200711155

[ORSP08] S. Okada, W. J. Ricker, and E. Sánchez Pérez, Optimal domain and integral extension of operators acting in function spaces. Birkhäuser-Verlag, Basel, 2008.

[Pe58] A. Pełczyński, On the isomorphism of the spaces $m$ and $M$. Bull. Acad. Polon. Sci. Sér. Sci. Math. Astr. Phys. 6(1958), 695-696.

[Pe60] Projections in certain Banach spaces. Studia Math. 19(1960), 209-228. http://dx.doi.org/10.4064/sm-19-2-209-228

[Ru80] J. B. Rutickil̆, Operators with homogeneous kernels. Sibirsk. Mat. Zh. 21 (1980), no. 1, 153-160; English transl. in: Siberian Math. J. 21 (1980), no. 1, 113-118.

[Si94] G. Sinnamon, Spaces defined by the level function and their duals. Studia Math. 111(1994), no. 1, 19-52. http://dx.doi.org/10.4064/sm-111-1-19-52

[Si01] The level functions in rearrangement invariant spaces. Publ. Mat. 45(2001), no. 1, 175-198. http://dx.doi.org/10.5565/PUBLMAT_45101_08

[Si07] Monotonicity in Banach function spaces. In: Nonlinear analysis, function spaces and applications, NAFSA 8, vol. 8, Czech. Acad. Sci., Prague 2007, 204-240.

[Ta81] M. Talagrand, Dual Banach lattices and Banach lattices with the Radon-Nikodym property. Israel J. Math. 38(1981), 46-50. http://dx.doi.org/10.1007/BF02761847

[Ta55] K. Tandori, Über einen speziellen Banachschen Raum. Publ. Math. Debrecen 3(1954), 263-268 (1955). 
[Wn93] W. Wnuk, Banach lattices with properties of the Schur type-a survey. Confer. Sem. Mat. Univ. Bari 249(1993), 1-25.

[Wn99] Banach lattices with order continuous norms. Polish Scientific Publishers PWN, Warszawa, 1999.

Department of Mathematics and Mechanics, Samara State University, Acad. Pavlova 1, 443011 Samara, Russia, and Samara State Aerospace University (SSAU), Moskovskoye shosse 34, 443086, Samara, Russia e-mail: astash56@mail.ru

Institute of Mathematics, Poznań University of Technology, ul. Piotrowo 3a, 60-965 Poznań, Poland e-mail: klesnik@vp.pl

Department of Engineering Sciences and Mathematics, Luleå University of Technology, SE-971 87 Luleå, Sweden

e-mail: lech.maligranda@|tu.se 